\title{
Multi-scale modelling strategy for textile composites based on stochastic reinforcement geometry
}

\author{
Andy Vanaerschott,*, Brian N. Cox ${ }^{\mathrm{b}}$, Stepan V. Lomov ${ }^{\mathrm{c}}$, Dirk Vandepitte ${ }^{\mathrm{a}}$ \\ ${ }^{a}$ KU Leuven, Dept. of Mechanical Engineering, Leuven, Belgium \\ ${ }^{b}$ Arachne Consulting, Sherman Oaks, CA, USA \\ ${ }^{c} K U$ Leuven, Dept. of Materials Engineering, Leuven, Belgium
}

\begin{abstract}
The quality of high-performance composite structures is difficult to predict. Variability in the macroscopic performance is dominated by the spatial randomness in the geometrical characteristics of the reinforcement, especially for textile composites. This work provides a roadmap for generating realistic virtual textile specimens spanning multiple unit cells, which are required to perform high-fidelity simulations. First, the geometrical variability in the reinforcement structure is experimentally quantified on the meso- and macro-scale in terms of average trends, standard deviations and correlation lengths. Next, each reinforcement parameter is modelled by the sum of its average trend and its zero-mean deviations, which are both determined by analysing experiments. Virtual specimens are then created using advanced simulation techniques that match the experimental statistics. Depending on the nature of measured correlations, the simulation technique is either a Monte Carlo Markov Chain method, a cross-correlated Karhunen-Loève Series Expansion technique or a Fourier Transform method used in combination with a Markov Chain algorithm. In a last step, a virtual representation of the textile geometry is constructed in geometrical modelling software, such as the commercially available WiseTex software.

The multi-scale framework is validated using data for a carbon-epoxy $2 / 2$ twill woven composite produced by resin transfer moulding: the simulated tow deviations trends repli-
\end{abstract}

\footnotetext{
${ }^{*}$ Corresponding author. Department of Mechanical Engineering, KU Leuven, Celestijnenlaan 300, 3001 Heverlee, Belgium. Tel.:+32 16322514 ; fax:+32 16322838

Email address: andyvanaerschot@gmail.com (Andy Vanaerschot)
} 
cate the target statistics.

Keywords: Textile composites, Multi-scale modelling, Non-determinism, Probabilistic methods

\section{Introduction}

The advantages of composites structures are well known. Besides a lower weight for the same or an enhanced performance, composites enable energy efficiency for air, ground and water transport. However, the introduction of composites remains hampered by the relatively high cost of raw material and the uncertain quality of high-performance composite structures. The latter is especially important in the aviation industry, where process variability is indicated as one of the main reasons for the increase in development time and cost for structural components [1].

Sufficient methods are available for the characterisation of mechanical properties by experiments and numerical simulation. Yet, there is a lack of understanding of how and why the mechanical properties vary across a composite product. Due to the specific nature of each composite with its specific manufacturing process, scatter can be very pronounced which impedes a correct estimate of the quality of the composite component. This variability in the macroscopic performance is directly linked with variability in the internal structure and constituents at the lower scales.

Variability in the reinforcement structure is frequently omitted or only partially introduced in simulations $[2,3,4]$. For the specific case of textile composites, the reinforcement is adequately modelled by exploiting the hierarchical principle. Predictive models are constructed following a sequence from fibre, tow, textile, preform, to the final composite [5]. To represent its internal geometry, a periodic unit cell model is considered where tow path characteristics are computed based on deterministic inputs such as fibre mechanics, topology, tow dimensions (shape, width, height) and tow spacing. These unit cell descriptions are considered to be repetitive along the entire structure without any variation in the tow position, shape and dimension. However, physical samples do show randomness in the geo- 
metrical parameters within a single unit cell and between neighbouring unit cells; tow path descriptors are spatially distributed across the composite [6]. Realistic modelling of internal geometry must include local variations along each individual tow path.

Among the different strategies for simulating the randomness in composites using appropriate scaling techniques $[7,8,9,10]$, those that are most likely to lead to accurate predictions of the statistical distributions of composite properties are calibrated by experimental quantification of the material variability. Charmpis et al. [4] present an excellent discussion of how the Stochastic Finite Element Method (SFEM) [11, 12] might be improved if experimental data were used to define the random fields that are incorporated within element properties. The desired modelling procedure for textile composites consists of three main steps: (i) collection of material data to define the stochastic geometry of tows (the step of uncertainty quantification and characterisation), (ii) generation of virtual specimens that replicate the measured statistics of the stochastic tow geometry and (iii) formulation of a stochastic multi-scale modelling scheme by which macroscopic material properties, and the variability in those properties, are predicted from the stochastic tow properties. When the first step is missing, analysts are forced to make assumptions regarding the input required for the second and third steps, leading to questionable estimates of the limits of material properties. The more detail that is available in the characterisation of the stochastic material microstructure, such as local tow path centroid variations and scatter in cross-sectional dimension, the higher the possible fidelity of simulations of damage evolution. In particular, not only are the distributions of material characteristics at any point important, but data defining the correlations between material deviations at different points can also be essential to complete prediction of the performance of a component $[4,8,13]$.

This article describes a multi-scale framework to generate realistic representations of the reinforcement geometry, in which the variability of fibre positioning in a textile is characterised on different scales and fused into virtual specimens that span many unit cells (typically $10^{3}-10^{5}$ unit cells) while retaining details of stochastic variability of tow geometry within a single unit cell. The article starts with a general overview of existing uncertainty 
modelling techniques applied to composites, before proposing the multi-scale strategy in section 3. Section 4 discusses the experimental frameworks that are defined to characterise the scatter in the internal geometry over two scales: the short-range, i.e. the unit-cell scale, and the long-range, i.e. the sub-component scale, which incorporates large numbers of unit cells. Next, random instances of tow path parameters are produced that match the experimental data using a Markov Chain [14], Series Expansion [15] or a combination of Fourier analysis and the Markov Chain algorithm [16]. In a final step, virtual models of the entire composite geometry are constructed using the WiseTex software [17], which is a geometry processor for textile fabrics (section 6). Each step throughout the developed framework is demonstrated for a 2/2 twill woven carbon fibre reinforced epoxy composite for which experimental data are already reported in prior publications $[18,19]$.

\section{Overview of existing simulation techniques for textile composites}

Methods of simulating the effects of random microstructure on composite properties can generally be classified into "non-intrusive" and "intrusive" approaches, terminology which we borrow from the field of spectral methods for uncertainty quantification, with applications in, e.g., fluid dynamics [20].

In the context of simulating the performance of a composite material with stochastic reinforcement geometry, a non-intrusive method uses a deterministic representation of the reinforcement geometry (e.g., perfectly regular plies in a tape laminate or perfectly regular, periodic tows in a textile composite) and adds stochastic character by assigning material properties to individual material elements that are drawn from statistical distributions; whereas an intrusive method uses representations of the reinforcement geometry that are themselves stochastic. Both non-intrusive and intrusive formulations are often analyzed in a Monte Carlo framework [21], in which instantiations of random material properties (nonintrusive method), or of a random reinforcement geometry (intrusive method), are generated by invoking a pseudo-random number generator; many specimens are generated and the scatter in composite properties are computed $[22,23]$. Intrusive methods can generally 
be decomposed into three groups depending on the manner in which correlations between deviations in the reinforcement geometry are treated, as detailed below.

\subsection{Non-intrusive simulation techniques}

Non-intrusive simulation techniques introduce the consequences of variations in the reinforcement geometry without changing the existing, idealized reinforcement model, but by changing local material properties instead, to the degree expected given the imperfection in the reinforcement.

Non-intrusive methods can be effective in a top-down engineering strategy for predicting the scatter in many engineering properties, provided relatively rich experimental data are used to calibrate the embedded statistical distributions. However, in the absence of detailed representations of stochastic reinforcement geometry, the degree of variability to be assigned to material properties is unknown a priori and the ability to predict performance is correspondingly limited.

\subsection{Intrusive simulation techniques}

\subsubsection{Methods based on spatially uncorrelated deviations}

The first group of intrusive methods introduces random deviations into the positions or shapes of tows, thus creating a stochastic reinforcement geometry, but treats the deviations at different locations as statistically uncorrelated.

One example is a study of non-crimp 3-D woven composites, in which the positions of nominally straight warp or weft tows were varied in a stochastic geometrical model of the composite according to statistical distributions determined by analyzing images of specimens, as described in [24, 13].

Instead of using experimental data, the amplitude of deviations in tow positions, in models in which spatial correlations have been neglected, have also been calibrated by matching predicted macroscopic mechanical behaviour with experimental results $[25,26]$; and computed by simulating the effect of disruptive mechanical loads on an initially ideal preform. 


\subsubsection{Methods including correlations between the deviations at pairs of points along a single tow}

In the second group of intrusive methods, spatial correlations are introduced among the deviations from ideal tow geometry that exist at pairs of points, but only for points residing on the same tow. We refer to this class of correlations as "auto-correlations". Given this restriction, stochastic geometrical parameters can be considered as random fields generated by a stochastic process, terminology that implies the presence of a single continuous independent variable, which, in the present application, is the position along a tow. Three different treatments of such models can be distinguished in the literature:

- In cases where correlation data are lacking, assumptions need to be made on the properties of the stochastic process. Examples of such modelling of tow paths in textiles is found in [27] and [28], where parametric trigonometric functions are used to represent the amplitude, wavelength and/or phase of the tow path parameters that are varied randomly from point to point on a grid with a fixed interval.

- Where experimental correlation information is available, the stochastic fields can be calibrated to reproduce the correlation data. Realisations of such random fields for textile features are obtained by decomposition of the correlation matrix by the Cholesky factorisation [29] or using Principal Component Analysis [30, 31], in combination with a vector of independent standard Gaussian random variables. Of these approaches, the Principal Component Analysis is more efficient for highly correlated vectors [32], and minimizes the dimensionality of representations.

- Auto-correlated deviations can also be produced for tows within textiles without decomposing the correlation matrix by using the Monte Carlo method to instantiate a Markov Chain model [33,34]. Tow deviations are generated by marching sequentially from grid point to grid point along a tow path, using a probability transition matrix that can be calibrated using experimental data for tow statistics. A post-processing operation is required to remove unphysical spikes in the generated tow path, which 


\subsubsection{Methods including correlations between the deviations at pairs of points on different tows}

In the third group of intrusive methods, spatial correlations are introduced among the deviations from ideal tow geometry that exist at pairs of points on different tows. We refer to this class of correlations as "cross-correlations". In the presence of cross-correlations, stochastic processes, whether analyzed by correlation matrices or the Markov Chain methodology, are no longer an adequate representation. Techniques are needed that accurately simulate the correlations in all directions: along the tow and between neighbouring tows.

The simultaneous reproduction of the auto- and cross-correlation structure of a single uncertain parameter demands more advanced generation techniques developed in the field of stochastic mechanics. An overview of the adaptation of such probabilistic methods to the particular case of textile composites, which will be the focus of this paper, is as follows.

- To acquire realisations of cross-correlated stochastic functions using the Cholesky decomposition, an anisotropic correlation matrix must be defined that represents the correlation information both along and between the tow directions. This is determined by defining a multi-dimensional normal distribution which can be described by e.g. a two-dimensional (2-D) extension of the Ornstein-Uhlenbeck process, calibrated to match the required covariance matrix [29].

- A methodology developed by Vořechovský [31] permits realisations of cross-correlated random fields based on the Karhunen-Loève (K-L) Series Expansion. The procedure was originally used to model correlated material properties in concrete, but is generally applicable for modelling a set of stochastic fields that share an identical auto-correlation function and of which the cross-correlation can be defined by a crosscorrelation coefficient. When applied to textile structures, each tow can be represented by a single random field [15].

- Analysis of cross-correlations in textile structures has also been demonstrated by 
combining discrete Fourier Transform analysis with the Markov Chain algorithm. Tow packing density deviations (deviations that vary relatively rapidly perpendicular to the tow direction) are generated using random values of the amplitude and phase of each Fourier component taken from experimental distributions. Relatively slow variations of the Fourier coefficients along the tow direction are then analyzed using the Markov Chain algorithm for amplitudes and a random-walk algorithm for phases. An inverse Fourier transform provides a virtual specimen generator, recreating corresponding deviation values at all grid points $[16,35]$.

\section{Multi-scale strategy for representing reinforcement variability}

We address the measurement and theoretical description of variability in the geometry of textile reinforcement over different scales, culminating in algorithms that generate virtual woven specimens that replicate the statistics of experimental samples. In this work, randomness in the numerical models is considered at the meso-scale (or the unit-cell scale) and macro-level (or the sub-component scale); variability in the arrangement of fibres within individuals tows is not considered, although recent experimental analyses have appeared elsewhere $[36,37,38]$. The variability of each tow path is defined for the centroid coordinates $(x, y, z)$, tow aspect ratio $A R$, tow area $A$ and tow orientation $\theta$ of a tow's cross-section which experience shows to offer a reasonably complete description of woven tows in a number of cases that have been studied. Figure 1 presents an overview of the multi-scale framework, where three main steps can be distinguished in obtaining random representations:

\section{Collection and statistical analysis of experimental data}

(a) Characterisation of the short-range variability (meso-scale) using samples close in size to the unit cell.

(b) Characterisation of the long-range variability (macro-scale) using samples spanning many unit cells.

(c) Statistical analysis of the tow path and shape parameters in terms of average trends, standard deviation and correlation lengths. 


\section{Generating instantiations of stochastic textile reinforcement using multi-scale modelling}

(a) Replication of average trends in the experimental data.

(b) Generation of zero-mean deviations that match measured short- and long-range correlations along each tow path.

(c) Generation of zero-mean deviations that match measured short- and long-range correlations between pairs of tows.

\section{Construction of virtual specimens by geometrical modelling software}

(a) Generation of a non-stochastic geometrical model of the tow architecture using topological ordering rules and tow characteristics supplied by the preform manufacturer.

(b) Superposition of stochastic tow path and shape deviations on the non-stochastic geometric model.

(c) Meshing of the geometrical model for use in FE calculations and attribution of material properties to each computational element that are derived from fibre and matrix properties supplied by the manufacturer.

In the next sections, we detail the execution of these three steps, but we stop short of presenting simulations of textile composite performance. We do not pursue the question of how such virtual specimens can be used in high-fidelity simulations to explore the role of material randomness in damage evolution, but refer the reader to a survey of recent work on this topic in which the damage is represented as multiple discrete cracks [35]. The computational problem of generating stochastic virtual specimens is sufficiently new and challenging to be our sole focus here. We further restrict consideration to nominally periodic textiles. The question of how the techniques developed for periodic cases might be adapted to quantify randomness in non-periodic textile preforms, such as developed for integrallywoven structures $[39,40]$ with symmetry-breaking features (sandwiches, heat exchangers, airfoils, etc.), is left for future work. 


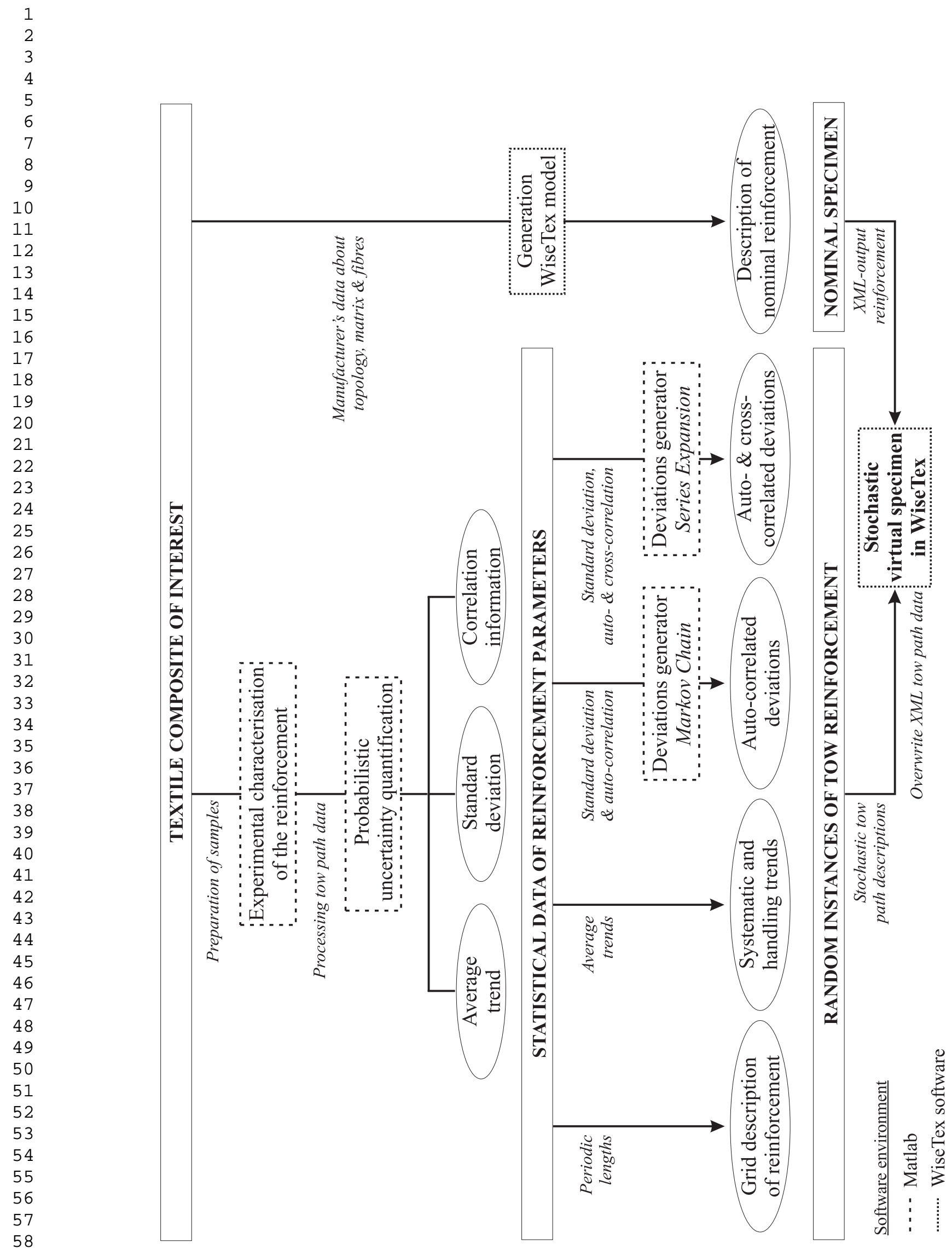

Figure 1: Multi-scale framework.

10 
All statistical analysis described below has been implemented in the Matlab application. The non-stochastic geometrical model of the preform (Step 3a) was generated using the commercially available WiseTex software package [17]. The examples describe a 2/2 twill weave of orthogonal warp and weft tows, which is a two-dimensional textile in the sense that it comprises a single relatively thin layer. When other topologies are considered, additional parameters enter the statistical analysis, e.g. the braid angle for braids and the distortion of the Z-yarn in case of three-dimensional non-crimp fabrics (e.g., angle interlock or orthogonal interlock).

\section{Collection and statistical analysis of experimental data (step 1)}

\subsection{Experimental framework}

\subsubsection{Experimental quantification of geometrical variation and global axis system}

Analysis of experimental data yields statistical metrics, which become the targets for reconstruction algorithms that generate stochastic virtual specimens: a large ensemble of instantiations of virtual specimens must have statistics that match the experimentally determined targets.

Geometrical variability arises within a single unit cell (short-range or meso-scale) and over many unit cells (long-range or macro-scale). Over the short range, 3-D geometrical information, including tow position and shape sampled over a grid with a grid spacing that is several times smaller than the unit cell length, can be collected using micron-resolution X-ray computed tomography (micro-CT). Over the long range, where much larger samples must be dealt with, geometrical information is commonly restricted to positional measurements, sampled over a relatively coarse grid, with spacing comparable to the tow width or to the unit cell size. Long-range positional measurements can be acquired for all tows using relatively low-resolution X-ray CT, or, for those tows that appear on the surface of the specimen, using optical imaging.

Data for tow path parameters from different tow paths can be separated according to the type or "genus" of the tow from which they originate, where tows of the same genus are 
nominally equivalent according to the definition of the textile architecture. For example, in the 2/2 twill weave analysed in section 4.2 and presented in figure 2, warp tows all belong to one genus, while weft tows belong to a second. In a 3-D weave, several distinct genuses of either warp or weft tows may exist [41, 33, 42].

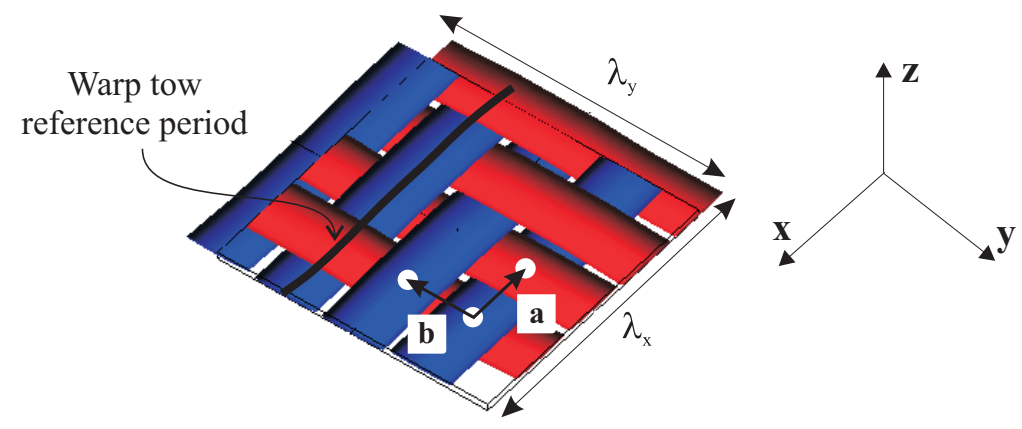

Figure 2: Illustrative WiseTex model of the weave architecture. The x-axis and y-axis of the coordinate system are respectively parallel to the warp and weft direction, in which the lattice vectors $(\mathbf{a}, \mathbf{b})$ are defined to collate data from tows of the same genus.

Data are further stored with reference to a global coordinate system. For woven textiles, we define a global coordinate system in which warp tows lie nominally in the $x$-direction, weft tows lie nominally in the $y$-direction, and the $z$-direction is the through-thickness direction (figures 2 and 3).

The directions of the coordinate axes are determined by finding the best fit of parallel straight lines to the data for all warp tows and all weft tows, subject to the restrictions that the set of lines fitting warp and weft tows must be orthogonal and the spacing for warp or weft tows of any genus must equal $\lambda_{x} / N_{g, y}$ or $\lambda_{y} / N_{g, x}$, respectively, where $\lambda_{x}$ and $\lambda_{y}$ are dimensions of the unit cell in warp and weft direction, respectively, and $\left\{N_{g, x}, N_{g, y}\right\}$ are the number of tows of a particular genus per unit cell in the warp and weft directions, respectively. This operation results in a hypothetical perfect lattice of straight, regularly spaced tows. Next, the actual position of any tow in a real or virtual specimen is defined relative to the corresponding straight line in the regular lattice. The lattice is discretised with uniform spacing which differs for either short-range or long-range data (figure 3). To analyse both the short- and long-range data, a system of four discrete rectangular 2-D lattices is gener- 
ated, comprising two relatively sparse grids for generating long-range positional variations for warp and weft tows (figure 4), and two relatively dense grids for generating short-range positional variations for warp and weft tows (figure 3 ). This is necessary since short-range and long-range variations are expected to arise from different sources. The largest wavelength deviations are created by handling of the fabric, and are therefore referred to as "handling-induced trends", whereas short-range wavelength deviations are believed to be generated by randomness in the motions of the weaving loom (mechanical chatter). The spacing of rows is set to the experimentally determined spacing for either warp or weft tows. The grid spacing along rows is either commensurate with the unit cell size (grids for long-range variations) or much smaller than the unit cell size (grids for short-range variations). It is convenient, although not necessary, to define grid locations so that the grid for long-range variations coincides with a subset of the grid for short-range variations.

For the $2 / 2$ twill weave in figure 2 , four tows of each genus occupy each unit cell $\left(N_{g, x}=\right.$ $N_{g, y}=4$ for both warp and weft tows) and the spacing of the tows of the same genus is uniform within the unit cell. For 3-D weaves where more than one genus of warp or weft tows are present, the offset of one genus from the other is allowed to vary in the fitting procedure and tows of different genuses may not be uniformly spaced within the unit cell [42]. However they are each separately uniform in their spacing over many unit cells.

\subsubsection{Statistical analysis}

In analyzing short-range experimental data (the unit-cell scale), advantage is taken of the expected periodicity of the textile. Each tow parameter characterizing position or shape for each tow genus can be decomposed into systematic, non-stochastic variations, which describe the average positional and shape variations imposed on a tow by the interlacing pattern of the weave over the domain of the unit cell, and stochastic deviations, which describe random departures from this average behavior. For parameter $\varepsilon \in\{\rho, z, A R, A, \theta\}$, with $\rho=y$ or $x$ for warp and weft tows, respectively, we write

$$
\varepsilon_{i}^{(j, t, s)}=<\varepsilon_{i}^{(j, t, s)}>+\epsilon_{i}^{(j, t, s)}
$$


where $\epsilon_{i}^{(j, t, s)}$ is the zero-mean deviation from the systematic value $<\varepsilon_{i}^{(j, t, s)}>$ at location $i$ $\left(i=1 . . N_{i}\right)$ along the tow $j\left(j=1 . . N_{g}\right)$ of tow genus $t$ in ply $s$. The label $s$ refers to the ply number, for cases where a laminate of stacked plies is considered. The systematic trends are identified using the method of "reference period collation" (figure 3) [41, 33]. Figure3(a) demonstrates the approach for two warp tows whose size is slightly larger than the unit cell. Figures 3(b) and 3(c) present the collation of all data for the tow genus onto a single reference period to determine the mean trend. Deviations in the short-range data are then defined by comparing the data for any tow at any location with the systematic trend curve at that position, which is created by translating the trend defined on the reference period through the appropriate lattice vector (figures 3(d)). While figure 3 exemplifies reference period collation for one positional coordinate, an exactly similar procedure can be defined for any tow characteristic.

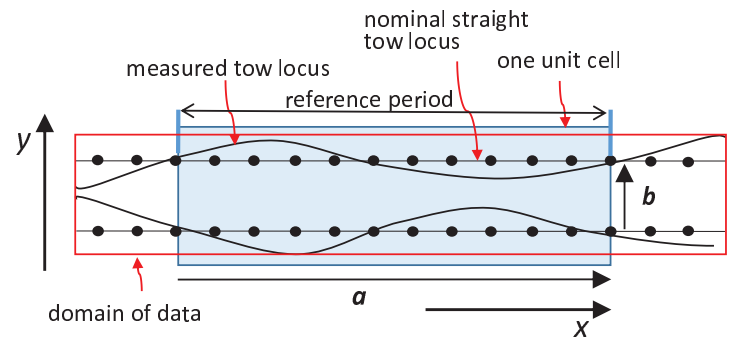

(a)

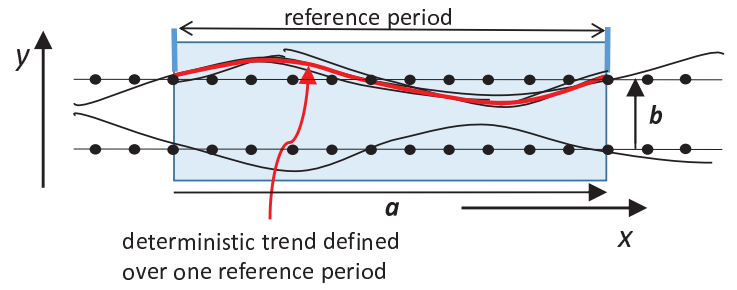

(c)

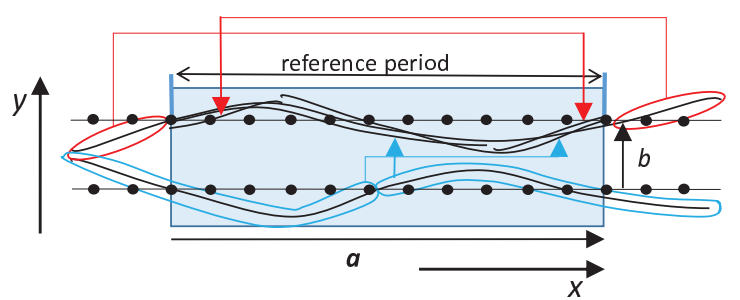

(b)

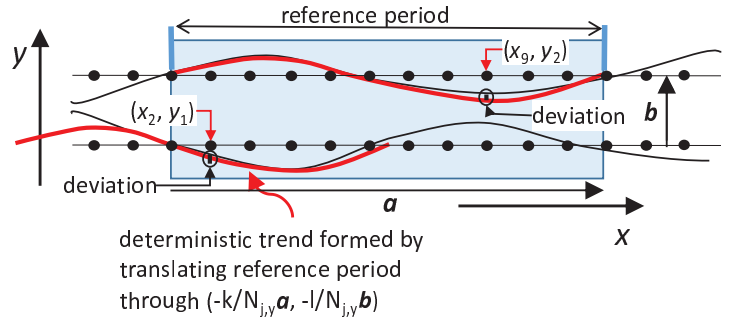

(d)

Figure 3: Reference period collation for short-range data, exemplified for the out-of-plane positional coordinate of tows in four steps: (a) data collection, (b) collation of data onto a single "reference period", (c) definition of systematic trend, (d) determination of the deviations from the systematic trend.

Long-range data sets have amplitudes tending to be much larger than those of deviations with shorter wavelengths, down to the unit cell size. The wavelength corresponds to what 
is expected if the specimen were to be gripped near its periphery and subjected to distorting loads, which is why we refer to these as "handling-induced trends". Handling-induced trends were deduced as illustrated in figures 4(a) and 4(b), by a procedure analogous to reference period collation, but with the reference period size equal to a specimen dimension. For this particular example, the positional data for each tow are translated in the $y$-direction so that they approximately coincide; and the mean of the superposed data is computed as the mean handling-induced trend.

With handling-induced trends thus defined to be uniform over the specimen, and effectively deterministic for that particular specimen, one may define stochastic deviations using an analogue of equation 1, i.e., decompose positional variations into a systematic trend (the handling-induced trend) and stochastic deviations. The definition of the stochastic deviations for long-range data is illustrated in figure 4(c) : the mean handling-induced trend is translated back to the nominal position of any tow $j$, and the difference between the data curve for that tow and the mean handling-induced trend at any grid point $x_{i}$ is recorded as the deviation in the tow position at grid point $\left(x_{i}, y_{j}\right)$. Handling-induced trends thus defined are deterministic for a single specimen, whereas the deviations of positions from the handling trends are stochastic within a single specimen. Handling-induced trends are generally expected to be stochastic over an ensemble of specimens.

\subsubsection{The data structure of deviations}

For both scales, the random deviations $\epsilon_{i}^{(j, t, s)}$ are computed for every data point on the grid as the departure from the mean behavior expected for that grid point for the systematic or handling-induced trends. The deviations constitute a "random field", defined over a 2-D spatial grid. Consider a tow parameter $\varepsilon \in\{\rho, z, A R, A, \theta\}$, with $\rho=y$ or $x$ for warp and weft

tows, that has been evaluated for one tow genus at each grid point (figure 3). The field of deviations in $\varepsilon$ over the entire specimen can be written as a $N_{i} \times N_{j}$ random matrix $\mathbf{H}$ :

$$
\mathbf{H}=\left\{\left[H^{1}\right]^{T},\left[H^{2}\right]^{T}, \ldots,\left[H^{N_{j}}\right]^{T}\right\}
$$




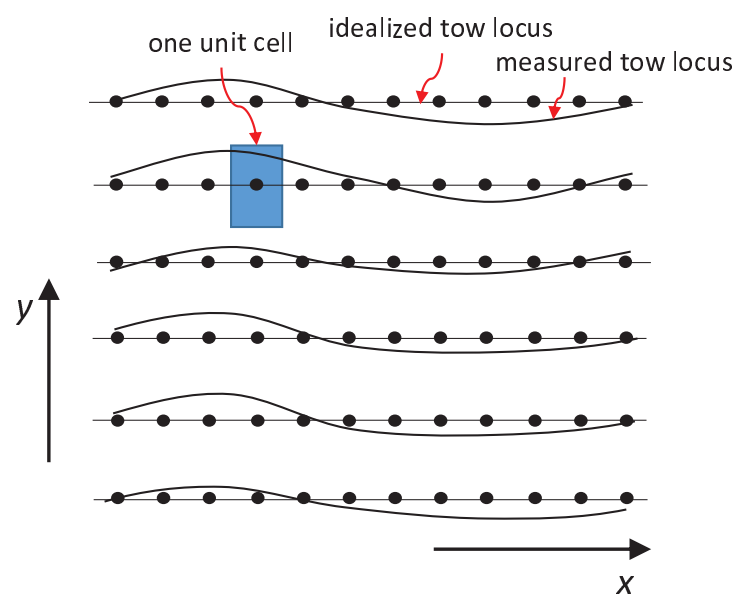

(a)

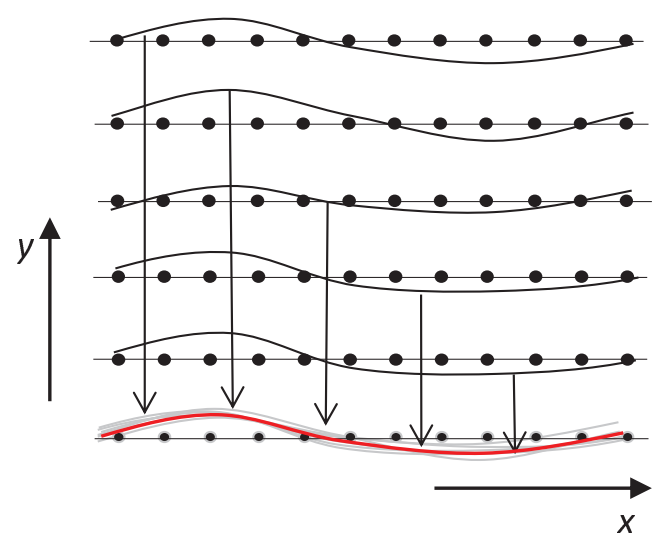

(b)

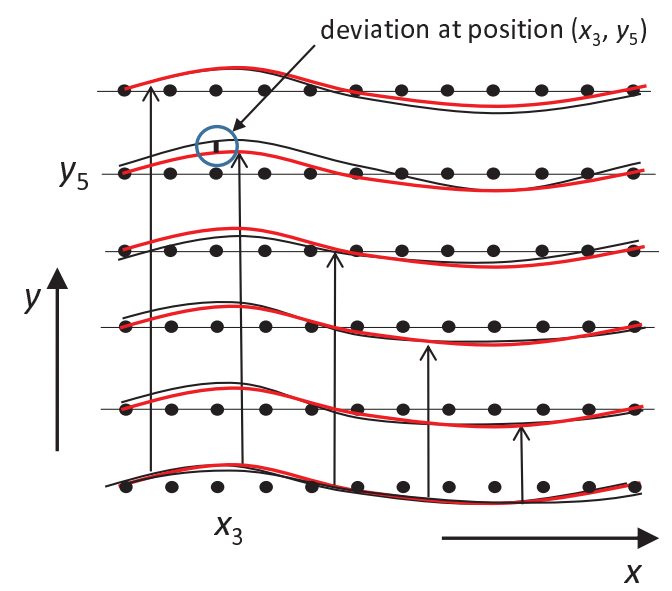

(c)

Figure 4: (a) Typical long-range data of positional variations, with (b) showing the procedure to define the handling-induced trends and (c) the operation to determine the deviations in the tow position due to machine noise.

with

$$
H^{j}=\left[\epsilon_{1}^{j}, \epsilon_{2}^{j}, \ldots, \epsilon_{N_{i}}^{j}\right]
$$

Each row in $\mathbf{H}$ corresponds to deviations (in position or geometrical characteristic) of a single tow which are defined at equidistant points along its length. For the example of inplane positional deviations of six adjacent warp tows, presented in figure 5, the $x$-direction contains $N_{i}$ grid locations, corresponding to the number of columns in $\mathbf{H}$, and the $y$-direction contains $N_{j}$ grid locations, corresponding to the number of rows in $\mathbf{H}$. 
Figure 5: In-plane positional deviations of six adjacent warp tows after subtracting the systematic or handlinginduced trend from the quantified in-plane positional positions. Deviations are defined on a rectangular grid, with the row spacing matched to the tow spacing. The red ellipses indicate relative positions for autocorrelation and cross-correlation for a spacing of 1 unit in the correlation matrix.

\subsubsection{Correlation information and matrix representation of correlations}

For both short-range and long-range data, correlation information is determined by computing Pearsons moment correlation coefficient for pairs of zero-mean deviation data (figure 5). In the following, we discuss the structure of correlation data involving a single tow parameter (e.g., one of the set $\{\rho, z, A, A R, \theta\}$ defined for short-range data) and a single tow type (warp or weft). Such analysis is valid when different types of tow parameters are uncorrelated. If correlations between the deviations in different tow parameters, or between warp and weft, are also considered, the data structure remains similar in form, but becomes much larger. Remarks on the size of correlation data sets appear in section 4.1.5.

First, we distinguish two categories of correlations, which recognize the fact that the magnitude of some correlations will be strongly influenced by the continuity of tows. "Autocorrelation" is determined by computing Pearson's correlation coefficient $C_{\text {auto }}(k)$ for pairs of data found for all grid points $i$ and $i^{\prime}$ that have the same spacing $k=i^{\prime}-i$ sampled on the same tow, repeated for all tows of the same genus. "Cross-correlation" is determined 
by computing Pearson's correlation coefficient $C_{c r o s s}\left(k, k^{\prime}\right)$ for pairs of data found at all grid points $(i, j)$ and $\left(i^{\prime}, j^{\prime}\right)$ that have the same vector spacing $\left(k, k^{\prime}\right)=(i, j)-\left(i^{\prime}, j^{\prime}\right)$ sampled on different tows $j$ and $j^{\prime}$, repeated for all pairs of tows of the same or different genus.

The correlation matrix $R_{\mathbf{H}}$ summarizes the Pearson's moment correlation coefficient information (both auto-correlations and cross-correlations) of any tow parameter. Most generally, one might define the correlation matrix as a square matrix of dimension $N_{j} N_{i} \times N_{j} N_{i}$, where $N_{i}$ is the number of grid points along a single tow (e.g., along $x$ in figure 5) and $N_{j}$ is the number of tows in the sample (e.g., number of grid points along $y$ in figure 5), with $N_{i}$ and $N_{j}$ taking the same values in each of an ensemble of equally sized, nominally equivalent specimens. To reflect the distinction of auto-correlations and cross-correlations, the correlation matrix $R_{\mathbf{H}}$ can be written as an array of $N_{j} \times N_{j}$ blocks with each block a submatrix $R_{j j^{\prime}}$ where the subscripts identify one of the $N_{F}$ tows of warp or weft type in the data set (e.g. tows 1 - 6 in figure 5)):

$$
R_{\mathbf{H}}=\left[\begin{array}{cccc}
R_{11} & R_{12} & \cdots & R_{1 N_{F}} \\
R_{21} & R_{22} & \cdots & R_{2 N_{F}} \\
\vdots & \vdots & \cdots & \vdots \\
R_{N_{F} 1} & R_{N_{F} 2} & \cdots & R_{N_{F} N_{F}}
\end{array}\right]
$$

Each submatrix entry is constructed by selecting one of the $N_{i} \times N_{i}$ correlation data values available for tows $H_{i}$ and $H_{j}$. Submatrix $R_{j j^{\prime}}$ is represented as:

$$
R_{j j^{\prime}}=\left[\begin{array}{cccc}
r_{j j^{\prime}}\left(x_{1}, x_{1}\right) & r_{j j^{\prime}}\left(x_{1}, x_{2}\right) & \cdots & r_{j j^{\prime}}\left(x_{1}, x_{N_{i}}\right) \\
r_{j j^{\prime}}\left(x_{2}, x_{1}\right) & r_{j j^{\prime}}\left(x_{2}, x_{2}\right) & \cdots & r_{j j^{\prime}}\left(x_{2}, x_{N_{i}}\right) \\
\vdots & \vdots & \cdots & \vdots \\
r_{j j^{\prime}}\left(x_{N_{i}}, x_{1}\right) & r_{j j^{\prime}}\left(x_{N_{i}}, x_{2}\right) & \cdots & r_{j j^{\prime}}\left(x_{N_{i}}, x_{N_{i}}\right)
\end{array}\right]
$$

with $r_{j j^{\prime}}\left(x_{i}, x_{i^{\prime}}\right)$ the Pearson's correlation value between deviation values at locations $x_{i}$ and $x_{i^{\prime}}$ on tow $j$ and tow $j^{\prime}$ respectively. Figure 5 highlights one pair of points separated in the $x$-direction that are needed to compute $r_{11}\left(x_{3}, x_{4}\right)$, and one pair of points separated in 
the $y$-direction needed to compute $r_{12}\left(x_{3}, x_{3}\right)$. The submatrices at the diagonal of $R_{\mathbf{H}}$ thus represent auto-correlations, while the off-diagonal entries are the cross-correlations between the different tow paths.

\subsubsection{Simplified representations of correlation}

As well as assuming the absence of correlations between different tow parameters, or between warp and weft pairs, a number of other assumptions about the nature of correlations are suggested by data that have been acquired to date $[41,18,19]$ :

\section{- Translational invariance}

It is often attractive to assume that the correlation between the deviations at a pair of grid points depends only on their vector separation $\tau_{x}=\left|x-x^{\prime}, y-y^{\prime}\right|$. If this is the case, then the number of degrees of freedom in the correlation matrix, $R_{\mathbf{H}}$, is greatly reduced.

\section{- Separable dependences of correlation on $x$ and $y$}

An even simpler structure in the correlation matrix arises if the correlation between the deviations at a pair of grid points shows translational invariance and can also be expressed as a separable function of the vector components $x-x^{\prime}$ and $y-y^{\prime}$. In this case, all the block matrices on any diagonal of $R_{\mathbf{H}}$, whether on the leading diagonal (auto-correlations) or off-diagonal (cross-correlations) become identical; and the elements on any row within any block matrix take values that depend only on the distance of that element from the lead diagonal of the block matrix, regardless of row. The block matrix $R_{j j^{\prime}}$ is now represented as:

$$
R_{j j^{\prime}}=\left[\begin{array}{cccc}
r_{j j^{\prime}}(0) & r_{j j^{\prime}}(1) & \cdots & r_{j j^{\prime}}\left(N_{i}-1\right) \\
r_{j j^{\prime}}(1) & r_{j j^{\prime}}(0) & \cdots & r_{j j^{\prime}}\left(N_{i}-2\right) \\
\vdots & \vdots & \cdots & \vdots \\
r_{j j^{\prime}}\left(N_{i}-1\right) & r_{j j^{\prime}}\left(N_{i}-2\right) & \cdots & r_{j j^{\prime}}(0)
\end{array}\right]
$$

with $r_{j j^{\prime}}(k)$ the Pearson's correlation value between deviation values of tow $j$ and tow 


\section{- Series expansions and correlation lengths}

An important characteristic of the correlation data matrix is the manner in which correlations decay as the distance between two grid points increases, which is, for the simplified matrix of equation 6 , the rate of decay of the magnitude of elements of any of the block matrices, $R_{j j^{\prime}}$, with distance of the element from the lead diagonal of $R_{\mathrm{H}}$. The most complete analysis of this decay is performed using a Series Expansion method. The Series Expansion method uses a modest number of degrees of freedom to describe the decay. In an alternative, simplest possible analysis, the decay is defined by a single parameter (a single correlation length). Both the Series Expansion method and the definition of the correlation length are described fully in section 5 .

The questions of how the functions $C_{\text {auto }}(k)$ and $C_{\text {cross }}\left(k, k^{\prime}\right)$ depend on the point separations $\delta x=x_{i+k}-x_{i}$ and $\delta y=y_{i+k^{\prime}}-y_{i}$ and whether cross-correlations $C_{\text {cross }}\left(k, k^{\prime}\right)$ are significant, determine the preferred method and the complexity of generating virtual specimens. More information on preferred reconstruction algorithms depending on the specific case is given in 5.2.

\subsection{Illustrative data from a 2/2 twill weave}

\subsubsection{Material description}

Data for a 2/2 twill woven carbon fibre Hexcel fabric (G0986 injectex) [43], impregnated with epoxy resin using a resin transfer moulding (RTM) production process [18], are used here for illustration. Interestingly, these data prove to be qualitatively similar to those found for a number of other weave types (3-D interlock weaves) for which data have been analyzed to date. Figure 2 shows a numerical model of the twill geometry with $\lambda_{x}=\lambda_{y}=11.43 \mathrm{~mm}$.

\subsubsection{Collection of short- and long-range information}

Short-range variations are identified in [18] from a seven-ply sample of dimensions comparable to one unit cell using laboratory X-ray micro-CT. Auto-correlations $C_{\text {auto }}(k)$ in 
the short-range data are characterized by a single scalar parameter, the correlation length $\xi$, which "measures the distance of two different stochastic field locations over which the correlation between the respective random variables approaches zero or a practically very small value" [4]. The value of $\xi$ is determined using linear regression of only the first five $k$ values, because higher $k$ values are noisy due to the small specimen size that can be analyzed by micro-CT. Cross-correlations in the short-range data are found by statistical analysis to be negligible, whether between tows of the same or different genuses [14].

Long-range data were acquired for the deviation of the in-plane centroid of each tow, sampled by optical imaging for a few locations per unit cell in large one-ply samples. Correlations among these data were analysed at two levels of detail. First, correlation lengths $\xi$ were deduced for decay along the tow and between tows (tables 2 and 3). The correlation length was evaluated for warp tows by fitting an exponential correlation function $C_{\text {exp }}=\exp \left(-\frac{\tau_{x}}{\xi}\right)$ to the data, while the correlation length between weft tows was evaluated by fitting a squared exponential correlation function $C_{s q, \exp }=\exp \left(-\frac{\tau_{x}^{2}}{\xi^{2}}\right)$ to the data.

Second, a fuller analysis of correlations was carried out using the eigenanalysis or Principal Component Analysis (PCA). Correlations were compiled exploiting the assumptions of translational invariance and separability defined above, leading to the form of equation 6 for the correlation matrix $R_{\mathbf{H}}$. The matrices for both warp and weft direction were constructed for a grid with $N_{i}=N_{j}=40$ and spanning a length of 10 unit cells by 10 unit cells. Under the assumptions of translational invariance and separability, each auto-correlation matrix entry corresponds to the Pearson's correlation value $r_{j j}\left(x_{i}, x_{i^{\prime}}\right)$ between deviation values at locations $x_{i}$ and $x_{i^{\prime}}$ on tow $j$, respectively, while the cross-correlation matrix considers Pearson's correlation value $r_{j j^{\prime}}\left(x_{i}, x_{i}\right)$ between deviation values at the same locations $x_{i}$ on tow $j$ and tow $j^{\prime}=j+k$ respectively, with $k=1 . . N_{j}-1$. Each entry is thus a coefficient or scalar (specified per k) that represents a single cross-correlation value valid for two fields that have an equal spacing equal (spaced by $k$ units).

The first ten eigenvalues (sorted from high to low) for PCA of the auto-correlation and cross-correlation matrices of the long-range in-plane positional deviations are shown in ta- 
ble 1 . As well as the eigenvalues, table 1 presents the normalised sum $\varrho$ as a measure of which eigenvalues are significant:

$$
\varrho(k)=\frac{\sum_{i=1}^{k} \lambda_{i}}{\sum_{i=1}^{N_{i}} \lambda_{i}}
$$

where $N_{i}$ the total number of eigenvalues generated and the $\lambda_{i}$ have been sorted into descending order. The parameter $\varrho(k)$ is used to choose the truncation point of series expansions in reconstruction procedures, as discussed in section 5.2.2.

Table 1: Ten first eigenvalues.

\begin{tabular}{ccccccccc}
\hline & $\begin{array}{c}\lambda_{\text {warp }}^{\text {auto }} \\
{[\mathrm{mm}]}\end{array}$ & $\begin{array}{c}\varrho_{\text {warp }}^{\text {auto }} \\
{[-]}\end{array}$ & $\begin{array}{c}\lambda_{\text {warp }}^{\text {cross }} \\
{[\mathrm{mm}]}\end{array}$ & $\begin{array}{c}\varrho_{\text {warp }}^{\text {cross }} \\
{[-]}\end{array}$ & $\begin{array}{c}\lambda_{\text {weft }}^{\text {auto }} \\
{[\mathrm{mm}]}\end{array}$ & $\begin{array}{c}\varrho_{\text {weft }}^{\text {auto }} \\
{[-]}\end{array}$ & $\begin{array}{c}\lambda_{\text {weft }}^{\text {cross }} \\
{[\mathrm{mm}]}\end{array}$ & $\begin{array}{c}\varrho_{\text {weft }}^{\text {cross }} \\
{[-]}\end{array}$ \\
\hline$\lambda_{1}$ & 0.334 & 0.739 & 3.224 & 0.081 & 9.451 & 0.625 & 7.911 & 0.198 \\
$\lambda_{2}$ & 0.062 & 0.877 & 3.107 & 0.158 & 4.220 & 0.904 & 7.296 & 0.380 \\
$\lambda_{3}$ & 0.020 & 0.922 & 2.929 & 0.231 & 1.186 & 0.982 & 6.377 & 0.540 \\
$\lambda_{4}$ & 0.097 & 0.944 & 2.711 & 0.299 & 0.230 & 0.998 & 5.283 & 0.672 \\
$\lambda_{5}$ & 0.006 & 0.956 & 2.475 & 0.361 & 0.033 & 1.000 & 4.151 & 0.775 \\
$\lambda_{6}$ & 0.004 & 0.964 & 2.237 & 0.417 & 0.004 & 1.000 & 3.095 & 0.853 \\
$\lambda_{7}$ & 0.003 & 0.970 & 2.009 & 0.467 & $3.64 \mathrm{e}-4$ & 1.000 & 2.191 & 0.908 \\
$\lambda_{8}$ & 0.002 & 0.974 & 1.799 & 0.512 & $2.95 \mathrm{e}-5$ & 1.000 & 1.474 & 0.944 \\
$\lambda_{9}$ & 0.001 & 0.977 & 1.610 & 0.553 & $2.07 \mathrm{e}-6$ & 1.000 & 0.943 & 0.968 \\
$\lambda_{10}$ & 0.001 & 0.980 & 1.442 & 0.589 & $1.28 \mathrm{e}-7$ & 1.000 & 0.574 & 0.982 \\
\hline
\end{tabular}

Table 1 implies that significantly different numbers of degrees of freedom will be required to represent the correlation along the tow direction and normal to the tow direction in reconstruction algorithms:

- eigenvalues for warp auto-correlations drop rapidly for both warp and weft tows, with the rate of decline significantly greater for weft tows.

- eigenvalues for weft auto-correlations drop relatively slowly, with the rate of decline significantly lower for the warp tows. 
Table 2: Standard deviation of the tow path parameters from the short-range [18] and long-range characterisation [19], respectively indicated by $s r$ and $l r$.

\begin{tabular}{ccccccccc}
\hline & $\begin{array}{c}\sigma_{x}^{s r} \\
{[\mathrm{~mm}]}\end{array}$ & $\begin{array}{c}\sigma_{y}^{s r} \\
{[\mathrm{~mm}]}\end{array}$ & $\begin{array}{c}\sigma_{z}^{s r} \\
{[\mathrm{~mm}]}\end{array}$ & $\begin{array}{c}\sigma_{A R}^{s r} \\
{[-]}\end{array}$ & $\begin{array}{c}\sigma_{A}^{s r} \\
{\left[\mathrm{~mm}^{2}\right]}\end{array}$ & $\begin{array}{c}\sigma_{\theta}^{s r} \\
{\left[{ }^{\circ}\right]}\end{array}$ & $\begin{array}{c}\sigma_{x}^{l r} \\
{[\mathrm{~mm}]}\end{array}$ & $\begin{array}{c}\sigma_{y}^{l r} \\
{[\mathrm{~mm}]}\end{array}$ \\
\hline$\sigma^{\text {warp }}$ & - & 0.113 & 0.014 & 1.774 & 0.023 & 0.797 & - & 0.106 \\
$\sigma^{\text {weft }}$ & 0.063 & - & 0.015 & 1.440 & 0.024 & 0.833 & 0.615 & - \\
\hline
\end{tabular}


(a)

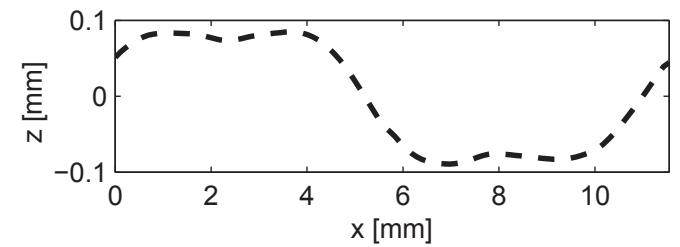

(b)

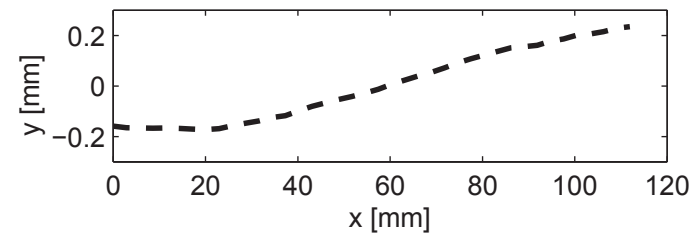

(c)

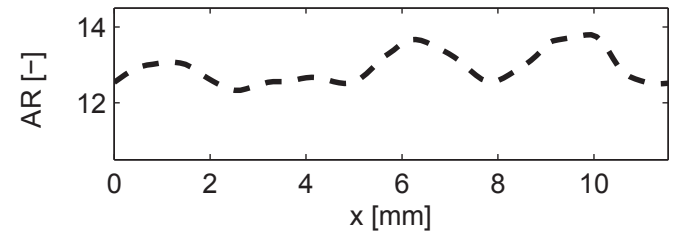

(d)

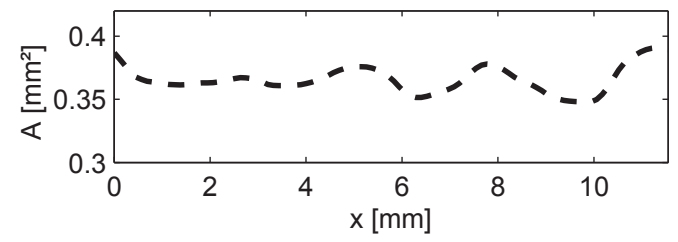

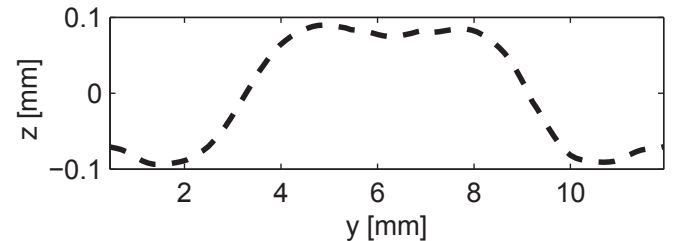
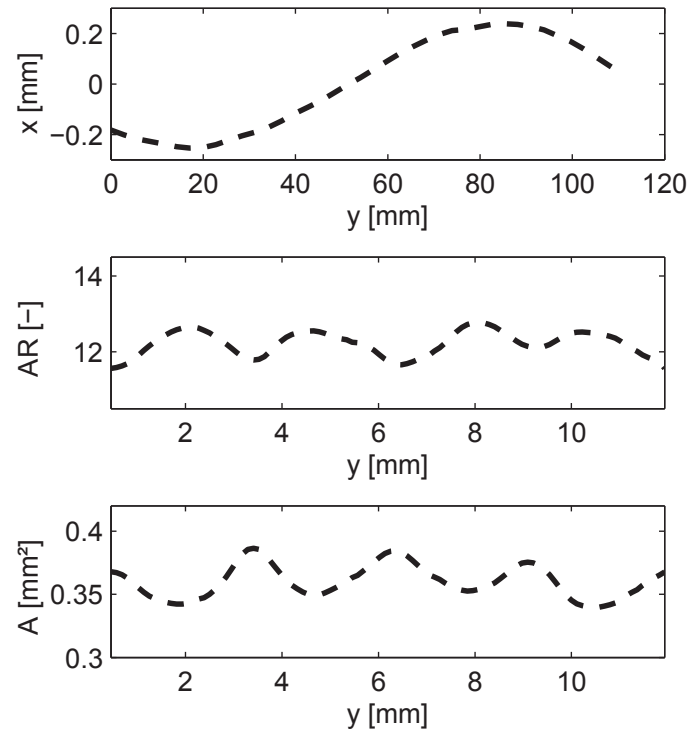

Figure 6: Periodic average and handling trends after translation of all tows per tow path parameter to their reference period, with the warp genuses defined along x-axis and the weft genuses along y-axis. Periodic trends are represented for one unit cell distance, while the handling effect is shown over a distance of ten unit cells.

Table 3: Correlation lengths of the tow path parameters from the short-range [18] and long-range characterisation [19], respectively indicated by $s r$ and $l r$. Only for the in-plane position a cross-correlation length is defined.

\begin{tabular}{ccccccccc}
\hline & $\begin{array}{c}\xi_{x}^{s r} \\
{[\mathrm{~mm}]}\end{array}$ & $\begin{array}{c}\xi_{y}^{s r} \\
{[\mathrm{~mm}]}\end{array}$ & $\begin{array}{c}\xi_{z}^{s r} \\
{[\mathrm{~mm}]}\end{array}$ & $\begin{array}{c}\xi_{A R}^{s r} \\
{[\mathrm{~mm}]}\end{array}$ & $\begin{array}{c}\xi_{A}^{s r} \\
{[\mathrm{~mm}]}\end{array}$ & $\begin{array}{c}\xi_{\theta}^{s r} \\
{[\mathrm{~mm}]}\end{array}$ & $\begin{array}{c}\xi_{x}^{l r} \\
{[\mathrm{~mm}]}\end{array}$ & $\begin{array}{c}\xi_{y}^{l r} \\
{[\mathrm{~mm}]}\end{array}$ \\
\hline$\xi_{\text {auto }}^{\text {warp }}$ & - & 22.89 & 1.78 & 7.26 & 2.53 & 4.56 & - & 114.89 \\
$\xi_{\text {cross }}^{\text {warp }}$ & - & - & - & - & - & - & - & 4.49 \\
$\xi_{\text {auto }}^{\text {weft }}$ & 9.42 & - & 1.62 & 5.48 & 1.01 & 3.49 & 52.89 & - \\
$\xi_{\text {croft }}^{\text {weft }}$ & - & - & - & - & - & - & 13.16 & - \\
\hline
\end{tabular}

\section{Generating instantiations of stochastic textile reinforcement using multi-scale mod- elling (step 2)}

\subsection{Generation strategy}

The strategy depicted in figure 1 yields ensembles of instantiations of stochastic textiles, or stochastic virtual specimens, by executing the following steps: 
1. A system of four discrete rectangular 2-D lattices is constructed for generating longrange positional variations and short-range positional variations for warp and weft tows. The grids can be, but are not necessarily, the same as those used to analyse data in section 4.1.1. Where archived statistics are being used to calibrate the virtual specimen generator, the grid used to analyse data may not even be known. It is also convenient to define the grid locations of the long-range lattice to coincide with a subset of the grid locations for the short-range variations. The grid spacing used for the short-range variations should be equal to or smaller than the shortest correlation length identified by experimental analysis. All components of long-range deviations are to be replicated in virtual specimens, then the overall dimensions of the virtual specimen (i.e., the total number of grid points) should exceed the longest correlation length identified by experimental analysis. The following steps are then iterated for each tow characteristic.

2. Long-range, handling-induced trends (figure 4) are generated for warp and weft tows at the grid locations of the sparser grid; values for these trends on the grid locations of the finer grid are then generated by interpolation.

3. Short-range, deterministic trends are generated for warp and weft tows at the grid locations of the finer grid. Since the virtual specimen is generally much larger than one unit cell, whereas the short-range, deterministic trends are recorded over a single reference period, the short-range, deterministic trends for the whole virtual specimen must be generated using the reverse of the process of reference period collation (figure 3) [33]: values at any grid point are set equal to the value at the corresponding point in the reference period, which is shifted from the grid point in question by a lattice vector, $k / N_{j, y} \mathbf{a}+l / N_{j, x} \mathbf{b}$, where $k$ and $l$ integers (section 4.1.2).

4. The long-range handling-induced trends and the short-range deterministic trends are combined at all locations on the finer grid by linear superposition. Linear superposition is valid because the spatial gradient in the long-range handling-induced trends is generally small compared to gauge lengths commensurate with the unit cell and there- 
fore the long-range handling-induced trends will not have influenced the deduction of short-range deterministic trends from experimental data.

5. Long-range stochastic deviations are generated at all locations of the sparser grid using Monte Carlo Markov Chain methods or Series Expansion techniques. Values of long-range stochastic deviations are then generated at all locations on the finer grid by interpolation.

6. Short-range stochastic deviations are generated at all locations of the finer grid using Monte Carlo Markov Chain methods.

7. The long-range deviations and the short-range deviations are combined at all locations on the finer grid by linear superposition. The validity of using linear superposition rests on whether the short-range and long-range deviations are uncorrelated. Because the characteristic wavelength of significant spectral components of the short-range deviations tends to be less than the shortest characteristic wavelength of long-range deviations, their correlation is likely to be weak. However, proof of this assumption awaits richer data sets.

8. The deterministic trends resulting from step (4) are combined with the stochastic deviations resulting from step (7) to create the complete specification of one tow characteristic throughout a single stochastic virtual specimen.

9. Steps (2) through (8) are repeated for all tow characteristics, and for both warp and weft tows, to create a single virtual specimen. Where no long-range data are available for a tow characteristic, the contribution from steps 2 and 5 are null. In the illustrative data in this paper, this is the case for all but the in-plane positional parameter. However, long-range out-of-plane positional deviations have already been reported elsewhere [16] and other characteristics may be measured in the future.

10. Steps (2) through (9) are iterated to generate an ensemble of stochastic virtual specimens.

We will illustrate this procedure, for a single ply of fabric with tow parameters consisting of the set $\{\rho, z, A, A R, \theta\}$. However, the procedure would remain the same for any type of 
periodic textile, including 3-D weaves, except that, if a larger numbers of tow genuses were present, the procedure would be based on an appropriately enriched lattice.

\subsection{Reconstruction algorithms}

A number of algorithms have been developed to solve the reconstruction problem of generating instantiations whose statistics match those derived from experimental data (standard deviation and correlation data). The algorithms were all developed to take advantage of the fact that statistical data for textiles tend, like the textiles themselves, to be highly anisotropic, usually with slow variations along the length of tows but rapid variations in the orthogonal direction. But even given this common condition, different algorithms are warranted depending on the richness of available data and differences in the magnitudes of certain correlations.

- A Markov chain algorithm deals with either short-range or long-range deviations along a single tow when auto-correlations decay in a simple manner or the experimental data are relatively sparse.

- A Series Expansion algorithm deals with either short-range or long-range deviations along a single tow when auto-correlations exhibit more complex behavior and the experimental data are relatively rich.

- A combination of Fourier analysis and the Markov Chain algorithm and a random walk model deals with long-range deviations in which cross-correlations are strong and complex (not well represented by a single correlation length), auto-correlations are strong but relatively simple (can be represented by a single correlation length for each amplitude and phase), and data are relatively rich.

- A matrix cross-correlated Series Expansion method deals with either short-range or long-range deviations where significant cross-correlations exist between tows, autocorrelation behavior is the same along each tow of any genus but relatively complex, 
and data are relatively rich. ${ }^{1}$.

In the following, we briefly describe the Markov Chain algorithm [33, 34, 18], the Series Expansion technique [31, 15], and the Fourier Transform method [16]. In section 5.3, we illustrate the advantages of the first two reconstruction algorithms (Markov Chain \& Series Expansion) by their application to data from the $2 / 2$ twill weave described in section 4.2.

\subsubsection{The Monte Carlo Markov Chain method for reconstructing auto-correlated devia- tions}

The Monte Carlo Markov Chain method offers a simple and fast method of reconstructing tow parameters for textile structures [33]. The algorithm is applicable when crosscorrelations of deviations on different tows are negligible. The Markov Chain generator acts for each tow parameter separately and is calibrated by the corresponding experimentally determined standard deviation and auto-correlation length.

First, the deviations of the considered parameter $\epsilon$ are represented by discrete values $\{-m a,-(m-1) a, \ldots, 0, \ldots,(m-1) a, m a\}$ with $m a=3 \sigma_{\epsilon}$. The parameter $m$ must be chosen not too low to avoid discontinuity errors due to the discreteness of the representation and not too high to minimize computational time. The probability of occurrence of the discrete values of $\epsilon$ constitutes the distribution vector $P_{i}^{\epsilon}$ for location $i$ :

$$
P_{i}^{\epsilon}=\left[\begin{array}{lllllll}
p_{m}^{(i)} & p_{m-1}^{(i)} & \ldots & p_{0}^{(i)} & \ldots & p_{-m+1}^{(i)} & p_{-m}^{(i)}
\end{array}\right]^{T}
$$

with $T$ denoting the transpose operation. The Markov process generates the distribution vector $P_{i+1}^{\epsilon}$ of the particular parameter $\epsilon$ at the next grid location $i+1$ using the probability transition matrix $A_{\text {trans }}^{\epsilon}$ :

$$
P_{i+1}^{\epsilon}=A_{\text {trans }}^{\epsilon} P_{i}^{\epsilon}
$$

In a pragmatic scheme, a tri-diagonal probability transition matrix (PTM) is constructed

\footnotetext{
${ }^{1}$ The requirement of identical auto-correlation behaviour is not mandatory, but considerably simplifies the analysis
} 
containing two independent parameters $\alpha$ and $\beta$ and a third dependent parameter $\gamma=1-$ $\alpha-\beta$ :

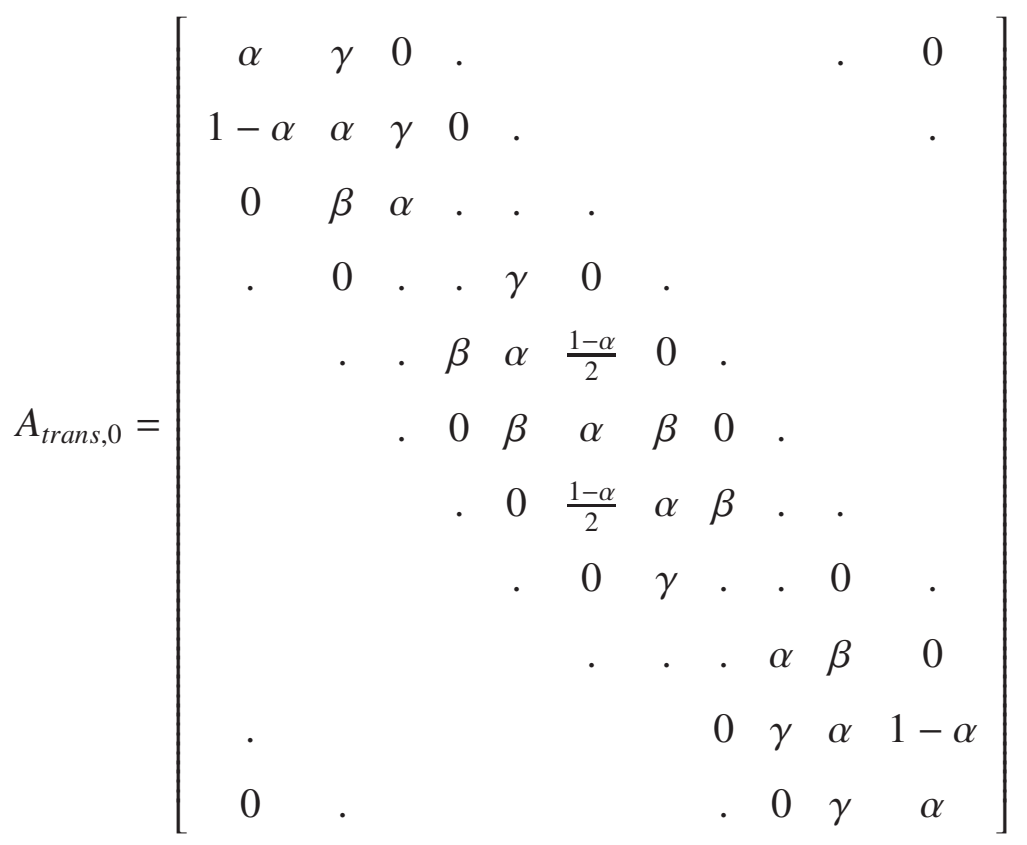

The parameter $\alpha$ is given an arbitrary fixed value of 0.9 , while $\beta$ is chosen to match the target standard deviation $\sigma^{\epsilon}$ (figure 7(a)): the more $\beta$ exceeds $\gamma$, the more likely is each application of the PTM to move the value of $\epsilon$ away from its mean. More information on choosing $\alpha, \beta \& \gamma$ can be found in [33]. Next, the target correlation length (information of the nearest neighbour: $k=1$ ) is matched by constructing the new PTM $A_{\text {trans }}^{\epsilon}=A_{\text {trans }, 0}^{N_{A}}$, which is the iterative re-application of the tri-diagonal form $A_{\text {trans }, 0}$. The parameter $N_{A}$ is calibrated from the graph shown in figure 7(b), which was built for the choice of $\alpha=0.9$, $m=10$ and a particular choice of $\beta$.

The Markovian procedure is the core computation within the Monte Carlo based scheme which is repeated for all parameters [33], with a different $(2 m+1)$ by $(2 m+1)$ probability transition matrix $A_{\text {trans }}^{\epsilon}$ for each tow parameter. A sequence of deviation values along a tow is initiated by mapping a uniform random number onto the cumulative probability distribution of the vector $P$ of equation 8 that represents the expected distribution of $A_{\text {trans }}$. Subsequent values in the sequence are generated by mapping further random numbers onto the row of $A_{\text {trans }}$ that corresponds to the value of the deviation $\epsilon$ at the previous grid location. 


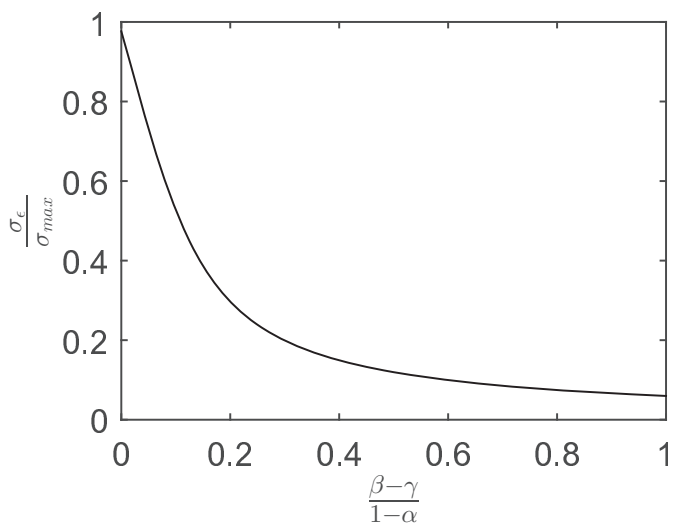

(a)

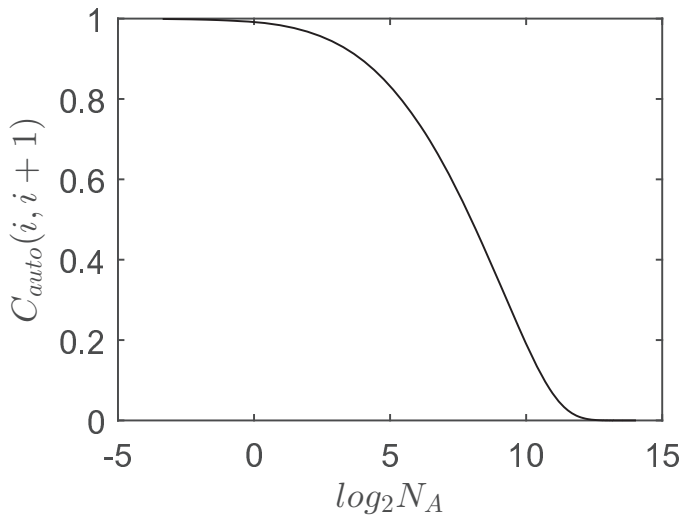

(b)

Figure 7: Graphs used to calibrate the probability transition matrix: (a) ratio $\sigma_{\epsilon} / \sigma_{\max }$, where $\sigma_{\max }$ is the variance generated by the PTM $A_{\text {trans }, 0}$ in the case $\beta=\gamma$ and (b) the number $N_{A}$ of iterative application of $A_{\text {trans }, 0}$ required to reproduce the target correlation length.

The generated deviations possess high-amplitude long-range wavelength fluctuations, according to the auto-correlation length, combined with low-amplitude short-range wavelength variations. The latter are numerical noise associated with the discreteness of the Markov Chain and are not observed in experiments. A post-processing smoothing operation reduces the short-range noise without affecting the statistics of the deviations to an unacceptable degree. The smoothing is a modified version of the moving box average that conserves the standard deviation [33]. Deviation values are typically smoothed using information over \pm 2 neighbouring grid points.

\subsubsection{The cross-correlated Karhunen-Loève Series Expansion technique for reconstructing auto- and cross-correlated deviations}

The K-L Series Expansion $[12,44]$ is a common technique to discretise and generate realisations of finite deviations represented as "random fields". This method involves the spectral representation of the correlation matrix $R_{\mathrm{H}}$, described in section 4.1.4, in a form of principal component analysis [45].

The following section describes (1) the general case, in which different tows of the same type may have different auto-correlation and cross-correlation between different tows of the 
same type are not necessarily spatially homogeneous, and (2) a simplified approach, for a specimen in which all different tows of the same type share the same auto-correlation behaviour and cross-correlations between different tows of the same type are spatially homogeneous, represented by a single coefficient.

Both algorithms are calibrated with the experimental standard deviation, and auto-correlation and cross-correlation statistics. Truncation of the principal component analysis in each correlation direction is different and related to how rapidly the variations occur: rapid variations in the direction normal to the tows and a much slower variation along the tow path, is the common case for long tows of continuous fibres.

In the general case (algorithm 1), a single realisation of a set of zero-mean tow path deviations $\left\{\tilde{\epsilon}_{i}^{j}, i=1 . . N_{i}, j=1 . . N_{j}\right\}$ in the same format as $\mathbf{H}$ and represented by $\tilde{\mathbf{H}}$, is generated by performing the Karhunen-Loève Series Expansion [46, 12, 44]:

$$
\tilde{\mathbf{H}}(x)=\sum_{z=1}^{N_{j} N_{i}} \sqrt{\lambda_{z}} \eta_{z} \phi_{z}(x)
$$

with $\left\{\eta_{z}, z=1 . . N_{j} N_{i}\right\}$ a set of centred orthonormal random variables and $\left\{\lambda_{z}, \phi_{z}, z=\right.$ $\left.1 . . N_{j} N_{i}\right\}$ the eigenvalues and eigenvectors obtained by solving the eigenequation of $R_{\mathbf{H}}$ :

$$
R_{\mathbf{H}} \Phi=\Lambda \Phi
$$

with $\Lambda$ a diagonal matrix with the $N_{j} N_{i}$ eigenvectors on its diagonal and $\Phi$ the eigenvector matrix that has as dimensions $N_{j} N_{i} \times N_{j} N_{i}$. In practice, a truncation of the series is performed. The number of K-L terms in the series is defined by ordering the eigenvalues in a descending series and considering only the larger eigenvalues, which correspond to the most influential variations. The number of eigenvalues retained is determined by testing for adequate representation of the experimentally measured randomness. Since these generated sets of deviations are standardised Gaussian random fields, an additional operation is performed to normalise the deviations by multiplication with the standard deviation of 
the distribution. The normalising factor is generally different for the same characteristic evaluated for tows belonging to a different genus: $H^{j} \sigma_{j}\left(j=1 . . N_{j}\right)$.

The general approach requires the solution of a high-dimensional eigenvalue problem of the correlation matrix $R_{\mathbf{H}}$ (order $N_{i} N_{j}$ ). Vor̆echovský (algorithm 2) proposes a significant reduction in this dimension for a set of deviations $\left\{\tilde{\epsilon}_{i}^{j}, i=1 . . N_{i}, j=1 . . N_{j}\right\}$, in which the correlation information of $R_{\mathbf{H}}$ can be fully represented by two separate correlation matrices: an auto-correlation matrix $R_{A}$ of order $N_{i}$, which is identical for all individual tow paths, and a cross-correlation matrix $R_{C}$ of order $N_{j}$ which describes all the cross-correlation coefficients between the different tow paths. These coefficients are scalars of which each represents the cross-correlation for all 1-D fields which have an equal spacing. Both matrices are constructed in a similar way as for the submatrix $R_{H_{j} H_{j}}$ in section 4.1 .4 while assuming that the correlation between the deviations at a pair of grid points depends only on their vector separation $\tau_{x}=\left|x-x^{\prime}\right|$ (section 4.1.5). Instead of performing a normalisation of the generated random fields to the experimental standard deviation, the auto-correlation matrix is already multiplied by the square of the standard deviation before performing its eigendecomposition.

The approach of Vořechovský based on $R_{A}$ and $R_{C}$ and using the Karhunen-Loève Series Expansion consists of subsequent steps to acquire $N_{\text {sim }}$ realisations of cross-correlated tow paths $\left(10^{4}\right.$ specimens $)[15,31]$ :

1. Perform eigendecomposition of the auto-correlation matrix $R_{A}=\left[\Phi^{A}\right]\left[\Lambda^{A}\right]\left[\Phi^{A}\right]^{T}$ and apply truncation: $\lambda_{i}^{A}, \phi_{i}^{A}(x)$ with $i=1 . . N_{v a r}\left(N_{v a r} \leq N_{i}\right)$.

Truncation is applied by ordering the eigenvalues $\lambda_{i}^{A}$ in a descending series and considering only the $N_{v a r}$ larger eigenvalues $\lambda_{i}^{A}$ and corresponding eigenvectors $\phi_{i}^{A}(x)$ that capture most of the randomness.

2. Perform eigendecomposition of the cross-correlation matrix $R_{C}=\left[\Phi^{C}\right]\left[\Lambda^{C}\right]\left[\Phi^{C}\right]^{T}$ and apply truncation: $\lambda_{i}^{C}, \phi_{i}^{C}(x)$ with $i=1 . . N_{f, r}\left(N_{f, r} \leq N_{j}\right)$.

Truncation is applied by ordering the eigenvalues $\lambda_{i}^{C}$ in a descending series and con- 

the same spectrum of eigenvalues $\lambda_{i}^{A}$ and -vectors $\phi_{i}^{A}(x)$, deduced from the auto-correlation matrix, but the sets of uncorrelated random variables used for the expansion of each field are now cross-correlated with neighbouring fields.

The introduction of cross-correlation for the random variables in step 4 is crucial in the procedure and reproduces the cross-correlation information between the different $N_{j}$ tow paths using the matrix decomposition method of equation 13. The eigenvalues $\lambda^{D}$ and vectors $\phi^{D}$ in equation 13 are solutions of the eigenvalue problem (similar as equation 12) 
of a block cross-correlation matrix $D$ of dimension $N_{v a r} N_{f, r} \times N_{v a r} N_{f, r}$ :

$$
D=\left[\begin{array}{ccccc}
I & C_{1,2} I & C_{1,3} I & \cdots & C_{1, N_{F}} I \\
\vdots & I & C_{2,3} I & \cdots & C_{2, N_{F}} I \\
\vdots & \vdots & I & \cdots & C_{3, N_{F}} I \\
\vdots & s y m & \cdots & \ddots & \vdots \\
\cdots & \cdots & \cdots & \cdots & I
\end{array}\right]
$$

This matrix possesses squared blocks $\left(N_{v a r} \cdot N_{v a r}\right)$ of unit matrices on its diagonal, while off-diagonal blocks represent the cross-correlation between each two sets of random fields using the entries of the cross-correlation matrix $R_{C}$.

In addition to this framework, Vořechovský proposes an improvement of the accuracy of the simulated auto-correlation by (i) applying correlation control techniques [47] and (ii) the anticipation of additional grid locations on the sides for each field. This first operation encounters the problem of spurious correlation which is sometimes introduced along the random variables $\chi_{j, i}^{D}$ of a single field. The effect of such techniques needs to be assessed for each new topology to determine if this computational expensive procedure is required or not. The operation of adding extra side points can be considered whenever disturbances are present in the generated values at the edges of the field.

\subsubsection{The Fourier Transform method for reconstructing auto- and cross-correlated devia- tions}

Whereas the Series Expansion method described above uses a single parameter to characterise cross-correlations, in some data sets cross-correlations, although extending over relatively short distances, exhibit relatively complex dependence on the separation of tows. Auto-correlations, although extending over long distances, exhibit relatively simple dependence on the separation of pairs of sampled points. For such cases, a method of Fourier analysis was developed that takes advantage of the anisotropy in the complexity of correlations to yield a relatively simple formulation with rapid execution [16]. The method is 
useful mainly for long-range data sets, extending over many unit cells.

Experimental data are of the type illustrated in section 4.2 for long-range variations for the twill weave, i.e., they consist of a 2-D grid of stochastic position values for one type of tow (warp or weft). The data are partitioned into a sequence of scan lines, oriented perpendicular to the nominal tow direction (figure 8). The position values along each scan line are subjected to discrete Fourier analysis, implemented using a Fast Fourier Transform (FFT) algorithm, which yields an amplitude and phase value for each frequency in a finite series. When the Fourier spectra for all scan lines are assembled, variations of the amplitude and phase of each spectral component along the nominal tow direction can be collated. For each amplitude, the variation along the tow direction is characterized by its standard deviation and correlation length. For each phase, the variation along the tow direction is modelled as a random walk and characterized by a mean path length. Thus the stochastic data for a given tow parameter are fully characterized by the standard deviation and correlation length of the amplitude, and the random walk mean path length of the phase, for each of a series of Fourier components. The number of Fourier components depends on the specimen size, but is typically 10 , to order of magnitude.

The generation of instantiations of the full 2-D field is executed by forming inverse Fourier transforms along each of the scan lines. The amplitude for each Fourier component to be used at each scan line in the sequence of scan lines is generated using the Markov Chain procedure of section 5.2.1, with the transition matrix $A_{\text {trans }}$ calibrated by the experimentally determined standard deviation and correlation length for that amplitude. The phase for each Fourier component to be used at each scan line in the sequence of scan lines is generated by a random walk generator, calibrated by the experimentally determined mean path length for that phase.

When the Fourier analysis method was applied to data for 3-D interlock weaves, handlinginduced deformation was revealed, similar to that described in section 4.2 for the twill weave data. A simple way of filtering such long-wavelength trends out of the Fourier analysis was demonstrated: if the Fourier analysis is applied to the spatial derivative of the position field, 
Figure 8: Data for positional variations of warp and weft tows in a 3-D angle interlock weave (from [16]). The images show spatial derivatives of the lateral positional variations of tows, which are dimensionless. Some representative scan lines are indicated (but not all), along which FFT analyses of the positional variations were performed. Variations of the Fourier coefficients from one scan line to the next can be described quite simply.

rather than to the field itself, the Fourier spectra are dominated by the shorter-wavelength spatial variations; the long-wavelength handling trends are filtered out.

\subsection{Application to a woven textile composite}

\subsubsection{Overview}

Virtual models are generated using the Markov Chain method and the Series Expansion method for the exemplary $2 / 2$ twill woven textile. Each virtual specimen spans a region of ten by ten unit cells, thus containing forty warp and forty weft tows. The model is representative of one ply within a laminate. Each tow is discretised in 320 equidistant points so that the information of one unit cell is defined over a grid of thirty-two points. This procedure is shown in figure 9 for the out-of-plane centroid coordinate. A total of $4 \times 10^{4}$ warp and weft tows, with lengths equal to ten times the unit cell periods, are simulated to create $10^{3}$ virtual specimens. Comparison with the experimental target values is performed by analysing histograms of the statistics and evaluating a normalised difference $\Delta$ from the 
target values, defined as

$$
\Delta=\left|\frac{v^{e x p}-v^{\operatorname{sim}}}{v^{\exp }}\right| \cdot 100 \%
$$

with $v$ equal to the standard deviation, auto-correlation length or cross-correlation length.

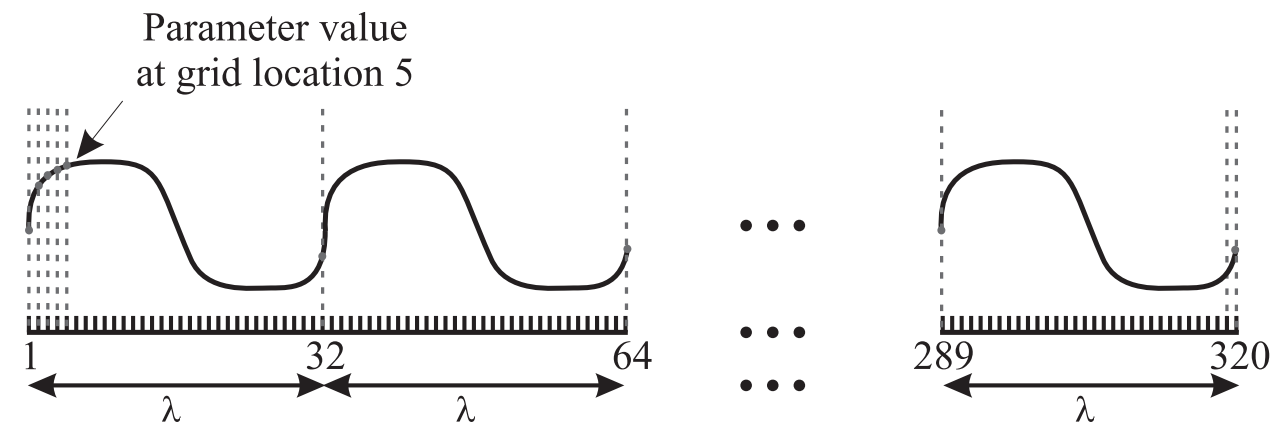

Figure 9: Procedure of generating a discretised tow representation, demonstrated for the out-of-plane centroid position.

The modelling procedure is based on several assumptions:

- Deviations are assumed to be normally distributed

- The cross-section of a tow is approximated by an ellipse

- Short-range and long-range deviations are statistically independent of each other

- In-plane long-range deviations, characterised from a single-ply sample, are also representative of a multi-ply sample

- Short-range deviations do not have any repetitive long-range effect exceeding the unit cell size

The first and second assumptions are validated by the limited experimental data for the twill weave, as well as data for 3-D woven ceramic matrix composites $[41,33,16,38]$. Validation for other materials, and for richer databases of the materials studied so far, awaits future experiments. A more realistic geometrical shape of a tow in cross-section can be acquired using moment analysis (see appendix A of [18]) or splines [48], without changing 
the nature of the generating procedure, only the number of parameters used to describe a tow. The lack of cross-correlation between short-range parameters and the long-range inplane centroid permits the independent generation of short-range and long-range deviations. The fourth assumption depends on inter-ply effects having a limited effect on the in-plane centroid path. The last assumption refers to the out-of-plane centroid, tow area and tow aspect ratio which are quantified from a unit cell sample. Although invalidation of this assumption would be surprising, additional quantification over longer-range samples would be reassuring.

\subsubsection{Average trends}

An average reinforcement description of the textile is acquired by (i) interpolation of the individual average periodic and handling trends of each tow path parameter to the equidistant grid locations over which the specimen is defined and (ii) combination of the different tendencies. Periodicity is exploited to construct the repetitive systematic trend of the shortrange parameters along the entire lattice. Figure 10 displays the average reinforcement of the $2 / 2$ twill woven composite.
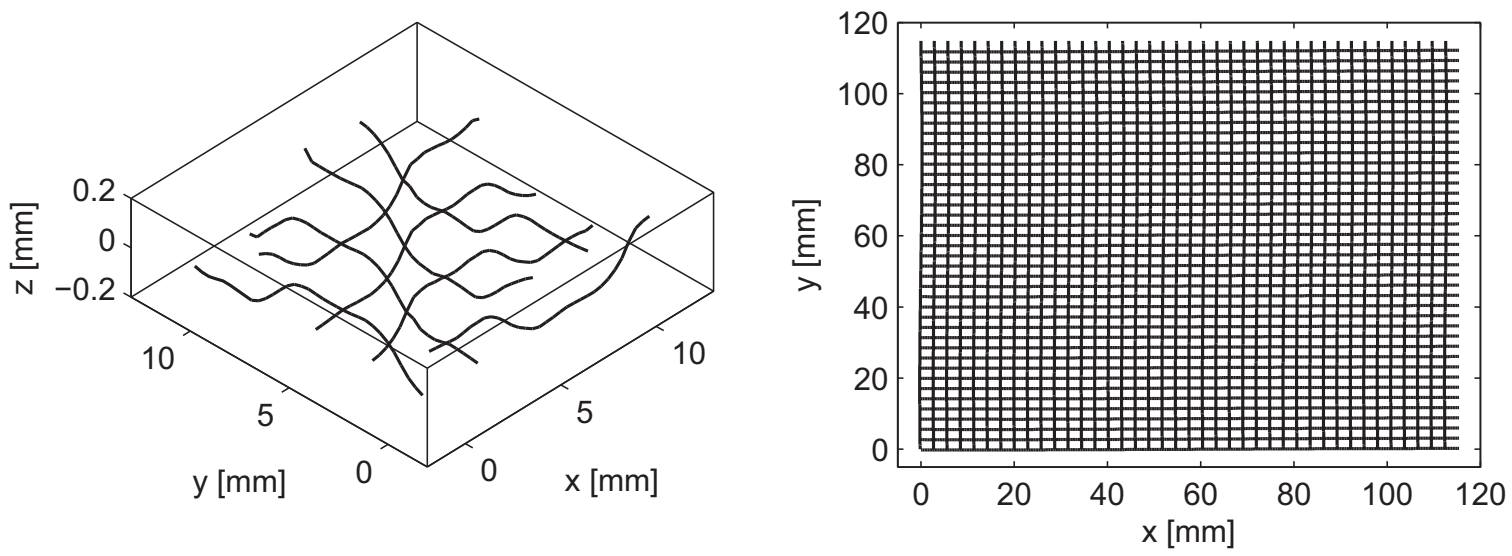

Figure 10: The average reinforcement description presented for the centroid coordinates at the short- and long-range, obtained by combining the average trends from figure 6 for all tow paths within the specimen. 


\subsubsection{Simulation of auto-correlated deviations by the Monte Carlo Markov Chain method}

All short-range deviations of the subjected 2/2 twill woven composite $\{z, A, A R, \theta\}$ are produced using this approach since for each of these tow path parameters no cross-correlation is observed while the data on correlation are not sufficiently rich to warrant a more elaborated method. It is sufficient to discretise the experimental deviations using twenty values $(m=10)$. As a post-processing step, a smoothing operation is performed using information of \pm 2 neighbouring grid points. The procedure is able to reproduce the wavelengths of fluctuations as demonstrated in figure 11 for the warp out-of-plane centroid coordinate.

A total of $4 \times 10^{4}$ warp and weft tows were created by the Markov Chain algorithm to construct $10^{3}$ long-range specimens. Reproduction of the experimental statistical data is demonstrated for the warp tows using (i) the combined data set, collecting the deviations of all $4 \times 10^{4}$ tows, and (ii) the data sets that represent all tows within single unit cells.

Figure 12 presents the auto-correlation graph of the experimental and simulated warp $z$-centroid for the combined data set. Calibration of $A_{\text {trans }}$ using only the nearest neighbours $(k=1)$ results in an exponential correlation course with minor fluctuations that crosses the zero-correlation boundary for large point spacings. The simulated standard deviation and correlation length for generated $z$-centroids and aspect ratio $A R$ are exemplified in table 4 .

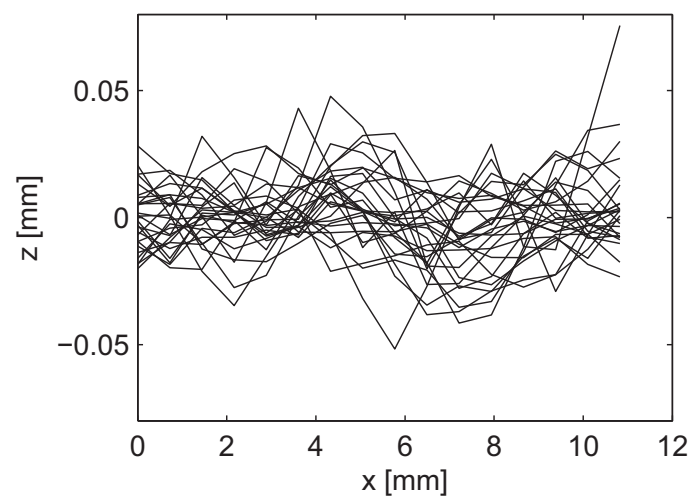

(a) Experiment

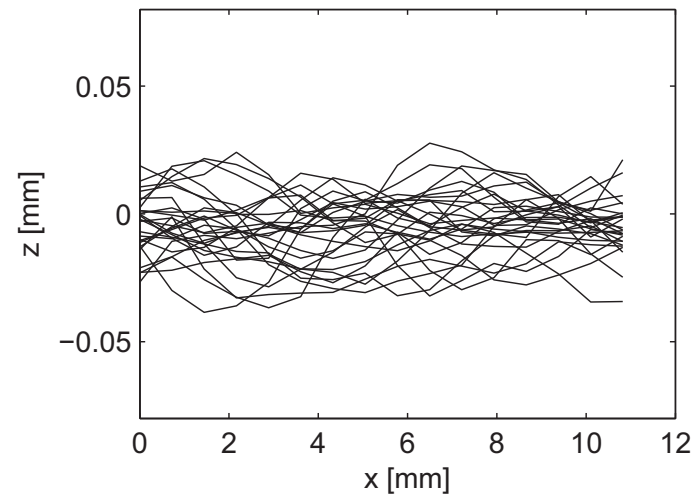

(b) Simulation

Figure 11: Out-of-plane centroid deviations trends for 28 warp tows: (a) experimental vs. (b) simulations (with smoothing). 
Standard deviations of all parameters are simulated with high accuracy $(\Delta<0.12 \%)$, while the correlation length of the tow path parameters of the $z$-centroid show significant normalised errors from the target data $(\Delta=73 \%)$. Some but not all of the normalised error $\Delta$ arises in the smoothing operation to remove short-wavelength noise: correlation lengths are slightly increased after the smoothing step, because neighbouring values are made more dependent.

Table 4: Standard deviation and auto-correlation length for the combined data set of warp $z$ and $A R$ deviations produced with the Markov Chain algorithm. Smoothed results are indicated by $s m$.

\begin{tabular}{lcccc}
\hline & $\begin{array}{c}\sigma_{z} \\
{[\mathrm{~mm}]}\end{array}$ & $\begin{array}{c}\sigma_{A R} \\
{[-]}\end{array}$ & $\begin{array}{c}\xi_{z} \\
{[\mathrm{~mm}]}\end{array}$ & $\begin{array}{c}\xi_{A R} \\
{[\mathrm{~mm}]}\end{array}$ \\
\hline Warp genus & 0.014 & 1.772 & 3.08 & 8.77 \\
$\Delta_{\text {warp }}$ & $0.11 \%$ & $0.12 \%$ & $73.16 \%$ & $20.90 \%$ \\
Warp genus - sm & 0.013 & 1.755 & 3.65 & 10.86 \\
$\Delta_{\text {warp-sm }}$ & $5.84 \%$ & $1.09 \%$ & $104.79 \%$ & $49.61 \%$ \\
\hline
\end{tabular}

In addition, the generated and experimental average unit cell standard deviation and auto-correlation length are compared. Unit cell statistics are computed by identification of tows belonging to a single unit cell in the virtual specimen. The Markov Chain permits a

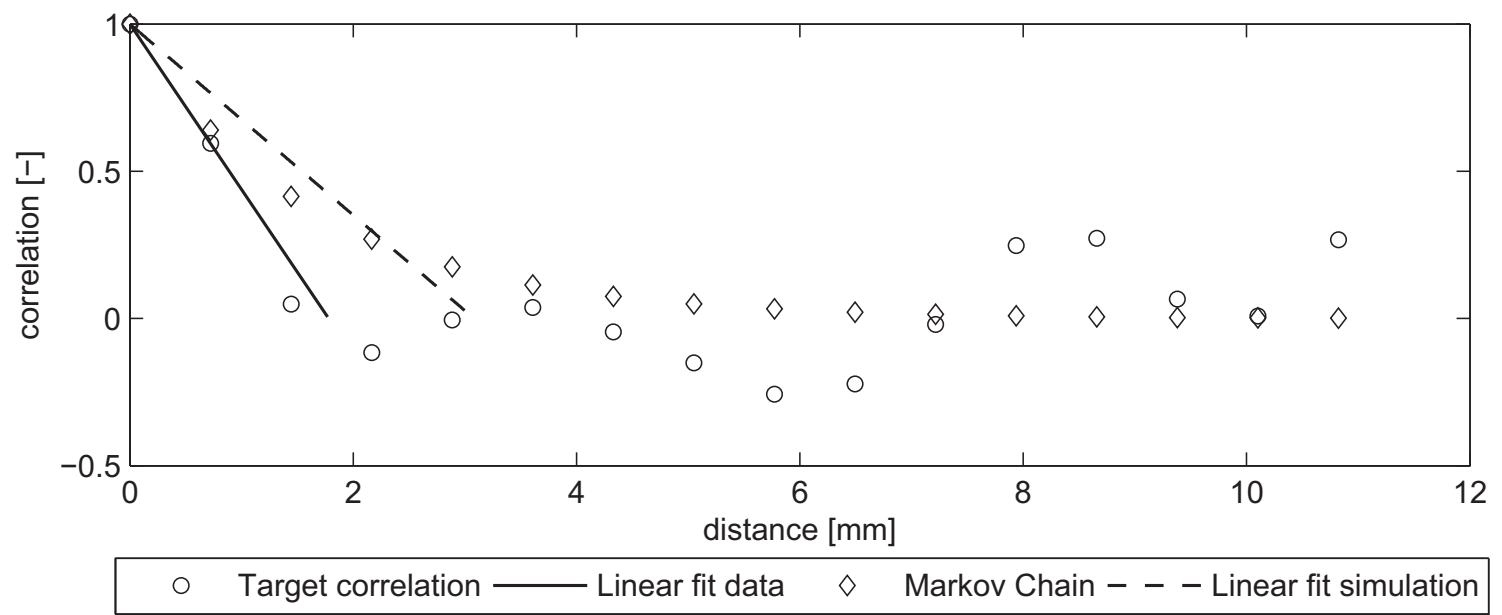

Figure 12: Correlation graph showing the experimental and simulated data of the warp $z$-centroid coordinate. A linear approximation of the first lag data is performed to deduce the correlation length. 
good comparison in average values for all short-range parameters as presented in table 5. The generated unit cell standard deviations and auto-correlation lengths are centred around the target values, for both warp and weft tows. This is demonstrated for the warp $z$-centroid coordinate in figure 13. Table 5 shows that smoothing has limited effect on the unit cell standard deviation (small increase in $\Delta$ ), while a high variance ( $\Delta$ of $50 \%$ till $73 \%$ ) is observed for the non-smoothed and smoothed correlation lengths.

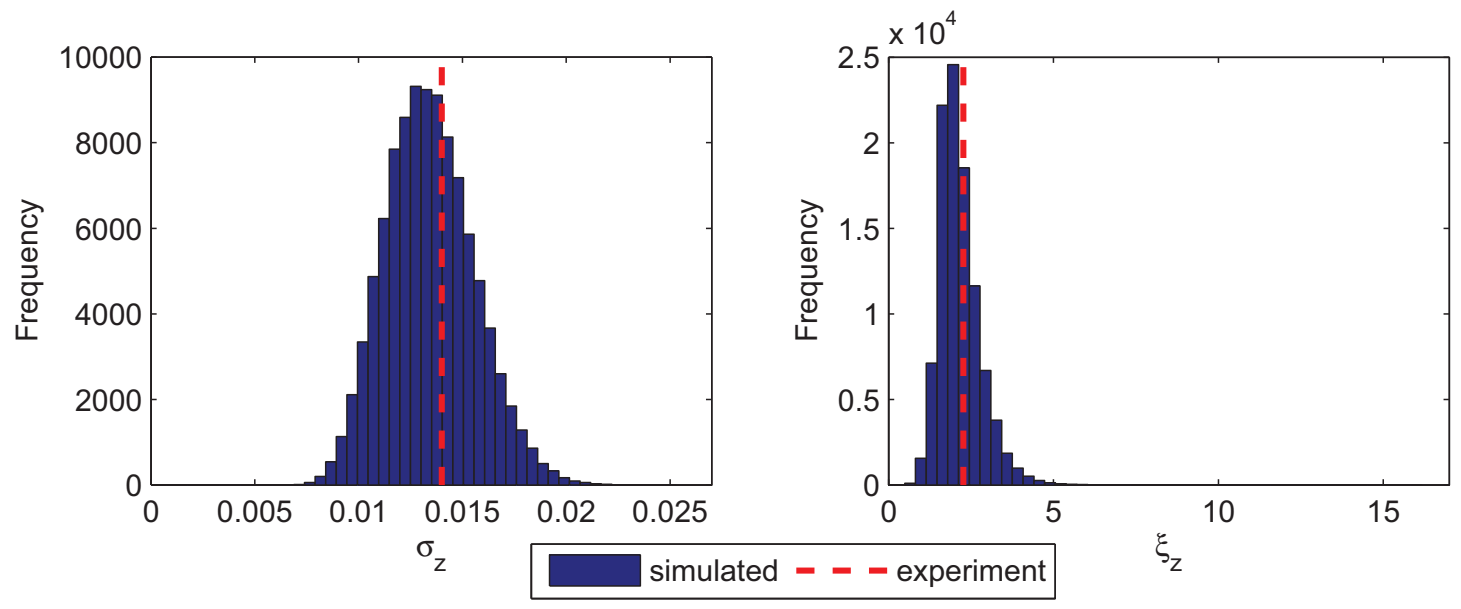

(a) Statistics of non-smoothed deviations

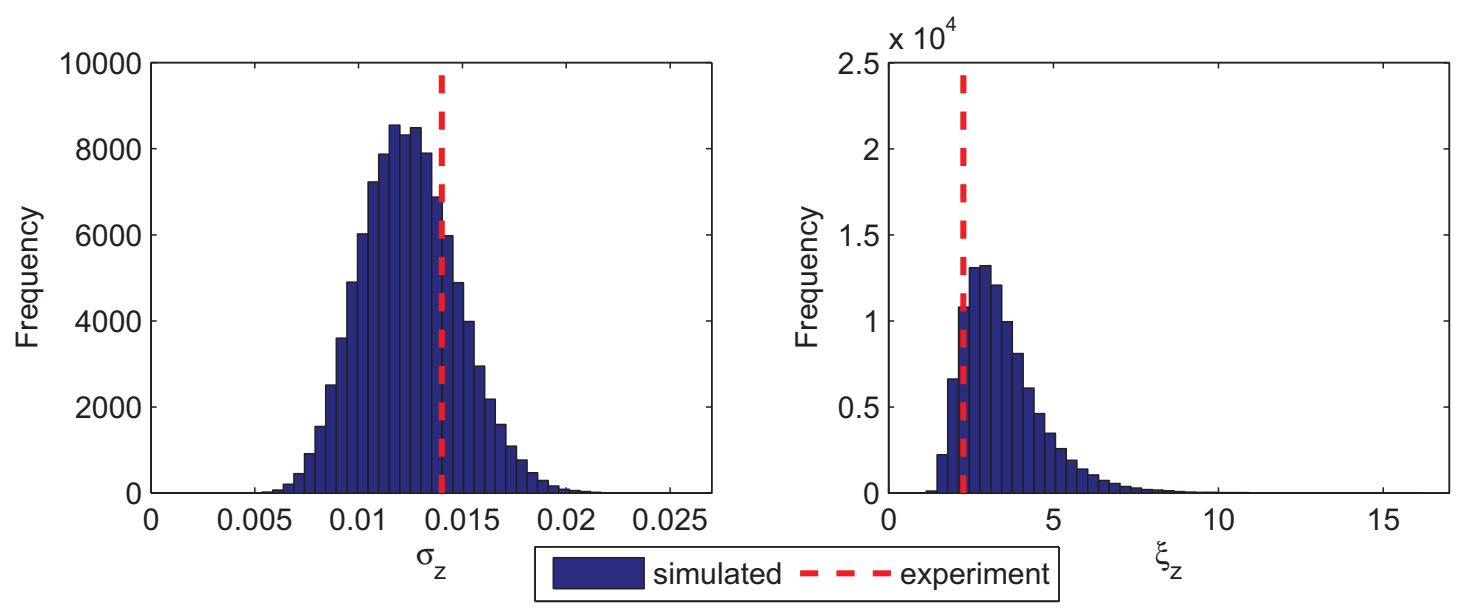

(b) Statistics of smoothed deviations

Figure 13: The unit cell statistics of the generated out-of-plane warp centroid positions (a) without and (b) with smoothing. Simulated data achieve the target statistics on average. When smoothing is applied, the simulated standard deviations are slightly affected, while all correlation lengths are increased. 


\subsubsection{Simulation of auto- and cross-correlated deviations by the cross-correlated Karhunen- Loève Series Expansion}

This technique is employed to simulate the in-plane centroid position $\{\rho\}$, which is the only tow path parameter in the considered 2/2 twill woven composite that exhibits a significant cross-correlation between adjacent tows. It is also the only parameter which possesses a long-range trend. The Series Expansion is performed separately for warp and weft genus with forty individual tows per genus in one specimen $\left(N_{j}=40\right)$, equidistantly spaced over ten times the periodic length in the $y$-direction for the warp genus or in the $x$-direction for the weft genus. Each set of long-range tow path deviations $\tilde{H}_{j}(x)$ is described over a sparse equidistant grid of forty-one points $\left(N_{i}=41\right)$ that span at least ten times the periodic length of the pertinent tow direction.

The auto-correlation and cross-correlation matrices are constructed from the fitted cor- 
relation functions to the computed Pearson's correlation values for different point spacings, deduced in section 4.2 and summarised in table 6. Auto-correlation is obtained by projecting the auto-correlation functions onto the grid of forty-one points representing the tow length, while the cross-correlation functions are projected onto the grid of forty points representing the in-plane positions of each individual tow path. In this procedure, it is assumed that the auto-correlation data only depend on the separation of points in the tow direction, while cross-correlation data depend only on the separation of points in the direction normal to the tows. In a last step, the generated long-range deviations, defined on a sparse grid of forty-one points, are interpolated at all locations on the finer grid of 320 points.

Table 6: Input correlation functions and applied truncation for simulating the in-plane fluctuations.

\begin{tabular}{lcc}
\hline & Warp tows & Weft tows \\
\hline Auto-correlation $C_{\text {auto }}$ & $\sigma_{w a}^{2} \exp \left(-\frac{\tau_{x}}{\xi_{\text {auto }}^{\text {warp }}}\right)$ & $\sigma_{\text {we }}^{2} \exp \left(-\frac{\tau_{x}^{2}}{\xi_{\text {aut }}^{2}}\right)$ \\
Cross-correlation $C_{\text {cross }}$ & $\exp \left(-\frac{\tau_{x}}{\xi_{\text {cross }}^{\text {vars }}}\right)$ & $\exp \left(-\frac{\tau_{x}^{2}}{\xi_{\text {croft }}^{\text {wefs }}}\right)$ \\
$N_{\text {var }}$ & 33 & 4 \\
$N_{f, r}$ & 40 & 13 \\
\hline
\end{tabular}

Realisations $\tilde{H}_{j}(x)$ of the in-plane centroids of warp and weft tows are computed using

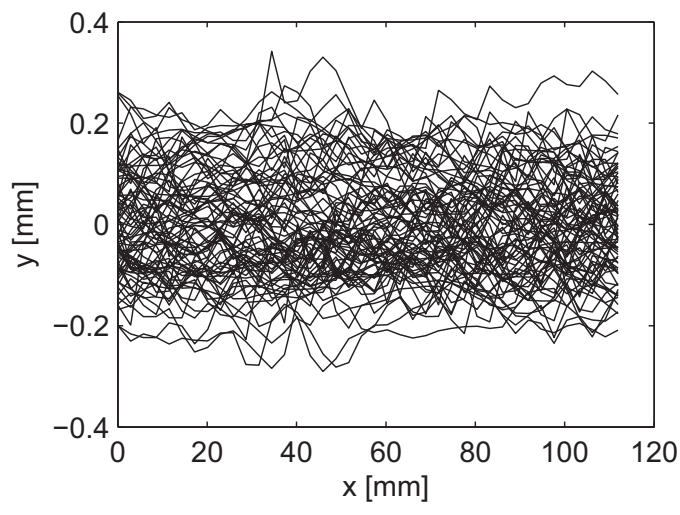

(a) Experiment

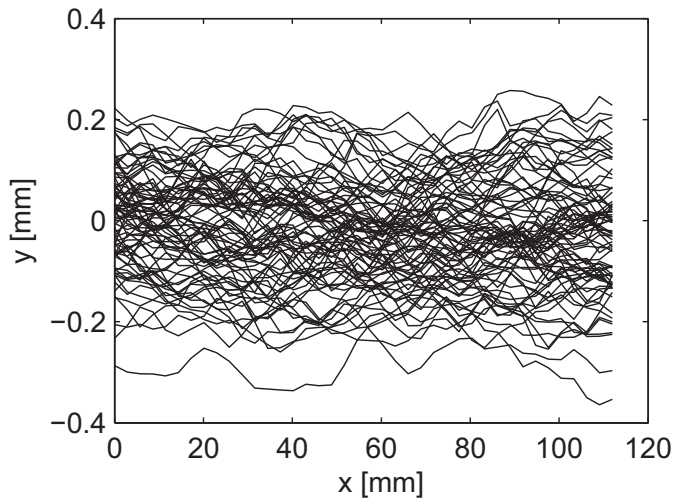

(b) Simulation

Figure 14: Warp in-plane centroid deviations trend for 80 warp tows: (a) experimental vs. (b) simulated deviations. 
the truncated series of equation 14. After sorting the eigenvalues, of which the ten largest eigenvalues are shown in table 1 in section 4.2.2, only the $N_{v a r}$ or $N_{f, r}$ largest eigenvalues and corresponding eigenvectors are considered in the procedure instead of $N_{i}$ and $N_{j}$, respectively. An appropriate measure of the captured variability is given by the normalised sum (or truncation error) $\varrho$ which is bounded below by 0.9975 :

$$
\varrho=\frac{\sum_{i=1}^{N_{\text {red }}} \lambda_{i}}{\sum_{i=1}^{N} \lambda_{i}} \geq 0.9975
$$

with $N_{\text {red }}$ equal to $N_{v a r}$ or $N_{f, r}$. The applied truncation for warp and weft deviations is related to wavelength of the variations in each direction and is given in table 6. Throughout the procedure, no correlation control techniques are considered to reduce possible spurious correlation between the random variables $\chi_{j, i}^{D}$. A sensitivity analysis concluded that this additional operation does not add in significant accuracy for the resulting statistics. Additional side points to the considered grid of the random field are not foreseen since no disturbance in the produced deviation values are observed at the beginning and end of the field.

In-plane centroid fluctuations are generated for $4 \times 10^{4}$ tows of each genus. The short wavelength of the experimental warp deviations and long wavelength of the measured weft variations are reproduced by the simulations. The correspondence for the warp in-plane deviation trend is demonstrated in figure 14. In contrast to the deviations produced with the Markov Chain algorithm in section 5.3.3, no additional smoothing operation is needed. Quantification of the spikes in the produced fluctuations concludes a less spiked path than observed in the experiments. This is not only attributed to the Series Expansion technique, but also to the normality assumption of the in-plane deviations which diminishes the presence of larger spikes in the simulated path.

The conformity between the experimental and simulated statistics is validated for the warp tows using data from one thousand generated virtual specimens. This information is investigated for the (i) combined data set, (ii) individual specimens consisting of hundred unit cells and (iii) individual 1-D fields, each representing one tow with a length of ten unit 
cells.

Statistics of the combined data set are precisely reproduced with normalised differences $\Delta$ for standard deviation $\sigma$ and correlation lengths $\xi_{\text {auto }}, \xi_{\text {cross }}$ which are less than $1 \%$ (table 7). Target correlation functions (table 6) and simulated correlation functions perfectly overlap with small differences for the largest lag spacings, shown in figure 15 for the warp auto- and cross-correlation graph.

When the statistical data per specimen $\left.(<\sigma\rangle,\left\langle\xi_{\text {auto }}\right\rangle,\left\langle\xi_{\text {cross }}\right\rangle\right)$ are considered, again good correspondence is obtained as indicated in table 7. The produced auto- and cross-correlation lengths for the warp tows of all thousand reinforcement descriptions are shown in figure 16. All generated correlation lengths have normalised errors $\Delta$ which are less than $3.48 \%$ for the standard deviation and maximum $1.72 \%$ for the correlation lengths.

Table 7: Standard deviation and correlation lengths for the (i) combined data set of in-plane positions and (ii) mean of the individual specimens, generated with the cross-correlated Series Expansion technique.

\begin{tabular}{lcccccc}
\hline & $\begin{array}{c}\sigma^{\text {comb }} \\
{[\mathrm{mm}]}\end{array}$ & $\begin{array}{c}\left\langle\sigma^{\text {spec }}\right. \\
{[\mathrm{mm}]}\end{array}$ & $\begin{array}{c}\xi_{\text {auto }}^{\text {comb }} \\
{[\mathrm{mm}]}\end{array}$ & $\begin{array}{c}\left\langle\xi_{\text {auto }}^{\text {spec }}\right. \\
{[\mathrm{mm}]}\end{array}$ & $\begin{array}{c}\xi_{\text {cross }}^{\text {comb }} \\
{[\mathrm{mm}]}\end{array}$ & $\begin{array}{c}<\xi_{\text {cross }}^{\text {spec }} \\
{[\mathrm{mm}]}\end{array}$ \\
\hline Warp tows & 0.106 & 0.103 & 115.81 & 114.00 & 4.54 & 4.42 \\
$\Delta_{\text {warp }}$ & $0.09 \%$ & $3.48 \%$ & $0.80 \%$ & $0.78 \%$ & $1.03 \%$ & $1.72 \%$ \\
\hline
\end{tabular}

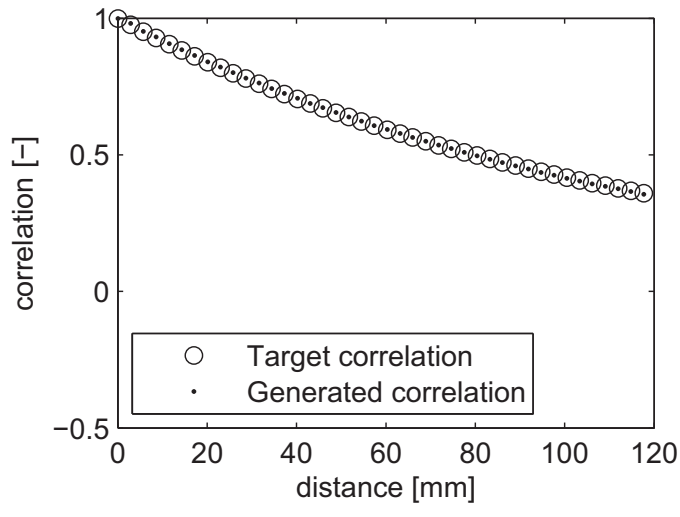

(a) Auto-correlation

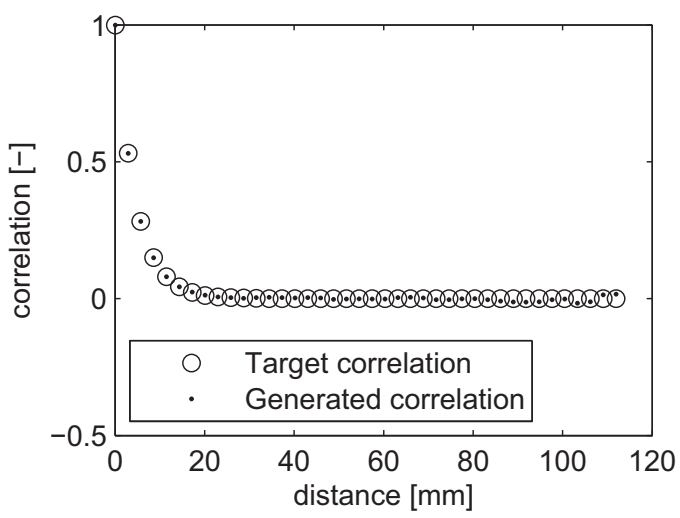

(b) Cross-correlation

Figure 15: Comparison of the warp input and simulated (a) auto-correlation and (b) cross-correlation functions. A perfect fit is obtained with minor fluctuations for the highest point spacings. 


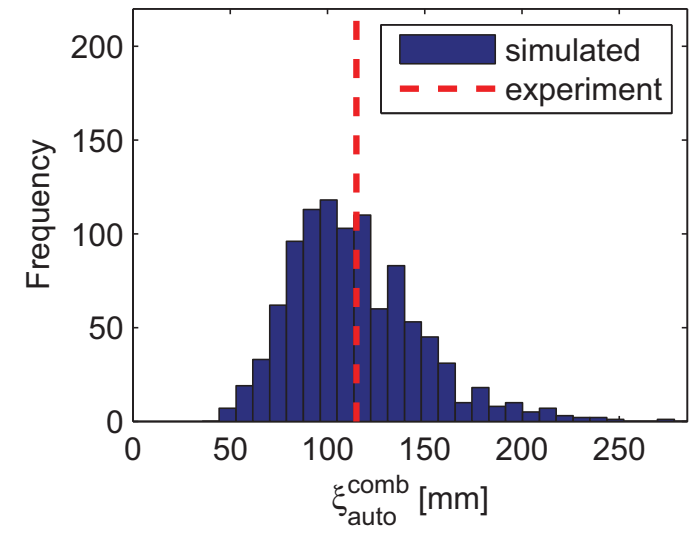

(a) Auto-correlation

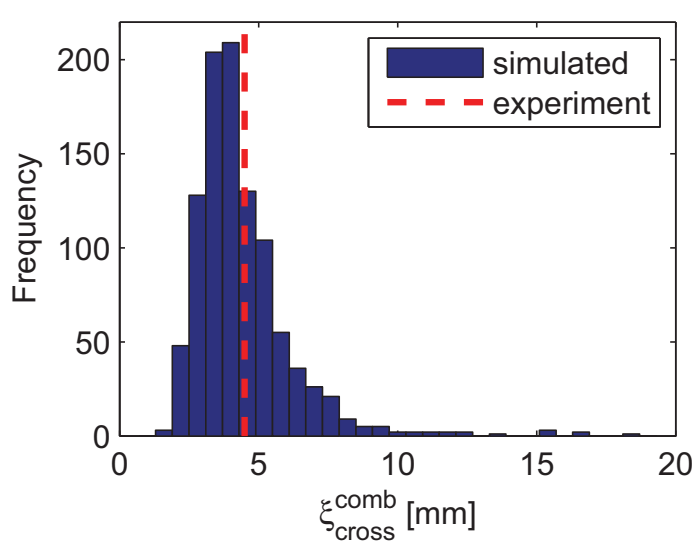

(b) Cross-correlation

Figure 16: Simulated (a) auto- and (b) cross-correlation lengths of the warp in-plane centroids. The experimental value is simulated on average.

An overview of the individual warp field statistics, in terms of standard deviation and auto-correlation length, is presented in table 8 . The experimental information of the individual tow standard deviation and correlation information is computed from arranging the data in [15]. While the generated standard deviation is obtained within 3\% error, the produced auto-correlation length only approximates the target value with similar order of magnitude. Latin Hypercube sampling of the independent deviations $\eta_{r}$ ensures a good similarity with the target mean and standard deviation. The difference in target and simulated correlation length is due to the normality assumption of the deviations distribution and the ideal fitted input correlation functions. These provoke the duplication of the statistical information on the individual tow level.

Table 8: Standard deviation and correlation lengths for individual 1-D random fields, representing the in-plane centroid, produced with the cross-correlated Series Expansion technique.

\begin{tabular}{lcccc}
\hline & $\begin{array}{c}\sigma^{\text {target }} \\
{[\mathrm{mm}]}\end{array}$ & $\begin{array}{c}\sigma^{1 D} \\
{[\mathrm{~mm}]}\end{array}$ & $\begin{array}{c}\xi_{\text {auto }}^{\text {target }} \\
{[\mathrm{mm}]}\end{array}$ & $\begin{array}{c}\xi_{\text {auto }}^{1 D} \\
{[\mathrm{~mm}]}\end{array}$ \\
\hline Warp tows - mean & 0.051 & 0.053 & 20.69 & 32.06 \\
$\Delta_{\text {warp,mean }}$ & - & $2.93 \%$ & - & $54.93 \%$ \\
\hline
\end{tabular}




\section{Construction of virtual specimens in a geometrical modelling software (step 3)}

The last step of the multi-scale framework (figure 1) creates virtual specimens with random geometry that possesses both long-range and short-range deviations with the appropriate auto-correlations and cross-correlations. In this paper, we exemplify this step by using the commercially available WiseTex software [17]. The tow path description of a nominal WiseTex model is overwritten by the stochastic tow path realisations, which are built as combination of the average trend with the generated zero-mean deviations for each tow path parameter. The WiseTex structure in Extensible Markup Language (XML) format is used to overwrite the tow path information since it permits scripting of local reinforcement information without the need of understanding the internal computational procedure [49]. In addition to the tow path description, other path information of the nominal model is updated such as the path length and the orientation vectors that fix each cross-section along the path in space $[14,50,5]$.

Figure 17 compares a nominal model with a generated stochastic specimen of the $2 / 2$ twill woven composite. A substantially different reinforcement description is observed. In contrast to the nominal model where warp and weft tows are following straight paths, the random model shows a significant difference in the in-plane centroid mobility for the warp and weft tows: weft tows are more variable and possess clustering behaviour between neighbouring tows. This results in long-range patterns of variation in the open spaces between neighbouring tows. The detailed image of an arbitrary unit cell shows the variation in the out-of-plane centroid position and tow cross-sectional variations.

\section{Discussion}

Generally, the statistics of a stochastic textile can be more completely replicated using methods that use all available auto-correlation information and incorporate cross-correlations as well. However, if all correlations are treated in full detail, the analysis can become intractably large. The three methods presented in this article offer different levels of approximation, each of which might be the preferred choice for data with given characteristics. The 
Figure 17: WiseTex representation of a nominal and stochastic virtual specimen. Warp and weft tows are respectively oriented horizontally and vertically.

Series Expansion method and the Fourier analysis method use a minimal representation of the spatial variation of correlations in one direction, while treating correlations in the other direction with detail. In the examples of their use presented here, the direction of minimal representation is perpendicular to the fiber direction in the Series Expansion method and parallel to the fiber direction in the Fourier analysis method. The Markov Chain method offers the simplest approach, using a minimal representation of correlations in the tow direction and ignoring correlations between tows. For data analysed to date, the simple concept of the transition matrix that underlies the Markov chain procedure has proven very appropriate for the spatially limited data available from micro-CT experiments $[41,33,18,14]$, where correlation information is limited and correlations are weak between different tows. In con- 
trast, the data acquired using optical imaging or digital image correlation cover much larger spatial ranges $[15,16]$, supporting the detail required by the Series Expansion or modified Fourier methods.

Fluctuations at the micro-level, such as fibre distribution and porosity inside a single tow, have not been treated here. Randomness in fibre positions within a tow has been measured using micro-CT imaging [36, 37, 38], but no reconstruction algorithm has yet been formulated. Micro-scale fluctuations are likely to be related to shape and positional deviations at higher scales, since the meandering interlacing of fibres with a stochastic bundle affects the constitutive behavior of the bundles [38] and therefore the deformation of a fabric.

In future work, the virtual specimens demonstrated here can be employed to predict the effect of geometrical variability on the macroscopic mechanical properties. The precise description of geometrical variability over the composite allows one to (i) predict its mechanical performance, such as stiffness, to define a quantitative measure of the spatial variation over the structure, (ii) perform damage simulations with a higher fidelity, or (iii) precisely simulate the resin impregnation of component-size fabrics. The random specimens in the WiseTex format, without any adjustments, are directly compatible with tools for micromechanical analysis such as stiffness evaluation $[23,5]$ and permeability simulations [51].

\section{Conclusions}

A generic multi-scale framework is developed to generate realistic virtual textile specimens. The aim of this approach is to deliver large textile models, i.e. consisting of multiple unit cells, with a reinforcement structure that possesses the same statistical information as quantified from the experimental samples. First, an experimental methodology is presented to characterise the geometrical variability in terms of the centroid coordinates and cross-sectional parameters on the short-range (meso-scale) and long-range (macro-scale). Non-destructive state-of-the-art inspection techniques such as X-ray micro-computed tomography, optical imaging or digital image correlation are applied to measure the fabric 
architecture in a reliable and efficient way across the composite volume. The inherent scatter of each tow path parameter in each tow direction is quantified in terms of an average trend, standard deviation and correlation information by applying the reference period collation method. For the data studied here, the correlations for long-range deviations in tow position constitute a 2-D random field over spatial dimensions $x$ and $y$. In a periodic textile, correlations are expected to exhibit translational invariance (dependence on the distances $\delta x$ and $\delta y$ between sampling points only). For the twill weave specimens, correlations were also found by analysis of the experimental data to be separable functions of $\delta x$ and $\delta y$, to a reasonable approximation. Furthermore, eigenanalysis of the correlation matrices shows that relatively few degrees of freedom are sufficient to model the decay of correlations with sampling point separation along tows, while considerably more degrees of freedom are required to model the decay in the direction normal to the tows. Yet, correlations persist over much longer distances along the tows than normal to them. Both these last results are in fact consistent with expectations of the effects of the continuity of tows.

Next, a stochastic multi-scale modelling approach is formulated, guided by the observations of translational invariance and separability and the results of eigenanalyses, to reproduce the measured variation in the tow reinforcement within the unit cell and between neighbouring unit cells. Random instances of tow paths are acquired by combining the deduced average trends with generated zero-mean fluctuations possessing the experimental standard deviation and correlation lengths on average. Zero-mean auto-correlated deviations are produced by the Monte Carlo Markov Chain for textile structures or a Series Expansion technique, while uncertain quantities that are dependent along and between tow paths (auto-correlated \& cross-correlated) are generated using a cross-correlated Series Expansion method or a Fourier Transform method in combination with a Markov Chain algorithm.

Last, virtual composite specimens with random fibre architecture are created in geometrical modelling software, such as the commercially available WiseTex software, using an intrusive approach. Nominal tow path descriptions are overwritten with stochastic tow 
representations, while preserving the original fibre mechanics and matrix properties. Concepts and procedures of this framework are developed for woven composites, but only minor modifications are required for other textile topologies.

The entire procedure is illustrated on a carbon-epoxy $2 / 2$ twill woven composite produced by RTM. Virtual specimens are simulated that span a region of ten by ten unit cells and are representative for a ply within a laminate. The experimental characterisation concludes that the geometrical variability of this high-performance textile is significant with substantial differences for warp and weft direction attributed to the manufacturing process of the weave. The in-plane coordinate is subjected to the largest variation exceeding the unit cell dimensions and is the only property of the tow path which is cross-correlated with neighbouring tows of the same type. Based on this information, deviations of the out-ofplane centroid $z$, aspect ratio $A R$ and area $A$ are produced using the Monte Carlo Markov Chain method, while the cross-correlated in-plane position $\rho$ is generated by the crosscorrelated Series Expansion procedure. A good comparison in terms of wavelengths and extreme values is obtained between the experimental and simulated deviations trends for all properties. Further, all simulated tow deviations achieve the target statistics on average.

\section{Acknowledgements}

This study is supported by the Flemish Government through the Agency for Innovation by Science and Technology in Flanders (IWT) and FWO-Vlaanderen. Brian N. Cox was supported by the US Army Research Office, Grant Number W911NF-13-1-0211.

\section{References}

[1] Warwick G. Qualifying carbon. Aviation Week \& Space Technology 2015;May.

[2] Cox B, Yang Q. In quest of virtual tests for structural composites. Science 2006;314(5802):1102-7.

[3] Graham-Brady L, Arwade S, Corr D, Gutierrez M, Breysse D, Grigoriu M, et al. Probability and materials: from nano- to macro-scale: a summary. Probabilistic Engineering Mechanics 2006;21(3):193-9. 
[4] Charmpis DC, Schuëller GI, Pellisetti MF. The need for linking micromechanics of materials with stochastic finite elements: A challenge for materials science. Computational Materials Science 2007;41(1):27-37.

[5] Lomov S, Huysmans G, Luo Y, Parnas R, Prodromou A, Verpoest I, et al. Textile composites: modelling strategies. Composites Part A 2001;32(10):1379-94.

[6] Mehrez L, Doostan A, Moens D, Vandepitte D. Stochastic identification of composite material properties from limited experimental databases, part 1: Experimental database construction. Mechanical Systems and Signal Processing 2012;27:471-83.

[7] Chamis C. Probabilistic simulation of multi-scale composite behaviour. Theoretical and applied facture mechanics 2004;41(1-3):51-61.

[8] Sriramula S, Chryssanthopoulos MK. Quantification of uncertainty modelling in stochastic analysis of FRP composites. Composites Part A 2009;40(11):1673-84.

[9] Kamiński M. Computational mechanics of composite materials: sensitivity, randomness and multiscale behaviour. London: Springer; 2005.

[10] Ostoja-Starzewski M. Microstructural randomness and scaling in mechanics of materials. London: Chapman \& Hall; 2008.

[11] Schuëller G. A state-of-the-art report on computational stochastic mechanics. Probabilistic Engineering Mechanics 1997;12(4):197-321.

[12] Ghanem R, Spanos P. Stochastic Finite Elements: a Spectral Approach. New York: Springer-Verlag; 2000.

[13] Dadkhah M, Flintoff J, Kniveton T, Cox B. Simple models for triaxially braided composites. Composites 1995;26(8):561-77.

[14] Vanaerschot A, Cox B, Lomov S, Vandepitte D. Stochastic multi-scale modelling of textile composites based on internal geometry variability. Computers \& Structures 2013;122:55-64.

[15] Vanaerschot A, Cox B, Lomov S, Vandepitte D. Simulation of the cross-correlated positions of in-plane tow centroids in textile composites based on experimental data. Composite structures 2014;116:75-83.

[16] Rossol M, Fast T, Marshall D, Cox B, Zok F. Characterizing in-plane geometrical variability in textile ceramic composites. Journal of the American Ceramic Society 2015;98(1):205-13.

[17] Verpoest I, Lomov SV. Virtual textile composites software wisetex: Integration with micro-mechanical, permeability and structural analysis. Composites Science and Technology 2005;65(15-16):2563-74. 
[18] Vanaerschot A, Cox B, Lomov S, Vandepitte D. Stochastic framework for quantifying the geometrical variability of laminated textile composites using micro-computed tomography. Composites Part A 2013;44:122-31.

[19] Vanaerschot A, Cox B, Lomov S, Vandepitte D. Stochastic characterisation of the in-plane tow centroid in textile composites to quantify the multi-scale variation in geometry. In: Proceedings of the IUTAM Symposium on Multiscale Modeling and Uncertainty Quantification of Materials and Structures. Santorini, Greece: Springer; 2014, p. 187-202.

[20] Maître OL, Knio O. Spectral methods for uncertainty quantification with applications to computational fluid dynamics. Springer; 2010.

[21] Metropolis N, Ulam S. The monte carlo method. Journal of the American Statistical Association 1949;24:335-41.

[22] Desplentere F, Lomov SV, Woerdeman DL, Verpoest I, Wevers M, Bogdanovich A. Micro-CT characterization of variability in 3D textile architecture. Composites Science and Technology 2005;65(13):1920-30.

[23] Olave M, Vanaerschot A, Lomov S, Vandepitte D. Internal geometry variability of two woven composites and related variability of the stiffness. Journal of Polymer Composites 2012;33(8):1335-50.

[24] Cox B, Dadkhah M. The macroscopic elasticity of 3D woven composites. Journal of Composite Materials 1995;29(6):785-819.

[25] Sejnoha M, Zeman J. Micromechanical modeling of imperfect textile composites. International Journal of Engineering Science 2008;46(6):513-26.

[26] Zeng X, Brown L, Endruweit A, Matveev M, Long A. Geometrical modelling of 3d woven reinforcements for polymer composites: prediction of fabric permeability and composite mechanical properties. Composites Part A 2014;56:150-60.

[27] Endruweit A, Long AC. Influence of stochastic variations in fibre spacing on the permeability of bi-directional textile fabrics. Composites Part A 2006;37(5):679-94.

[28] Abdiwi F, Harrison P, Koyama I, Yu W, Long A, Corriea N, et al. Characterising and modelling variability of tow orientation in engineering fabrics and textile composites. Composites Sciences and Technology 2012;72(9):1034-41.

[29] Skordos AA, Sutcliffe MPF. Stochastic simulation of woven composites forming. Composites Science and Technology 2008;68(1):283-96.

[30] Yushanov S, Bogdanovich A. Fiber waviness in textile composites and its stochastic modeling. Mechanics of Composite materials 2000;36(4):297-318. 
[31] Vořechovský M. Simulation of simply cross correlated random fields by series expansion methods. Structural Safety 2008;30(4):337-63.

[32] Phoon K, Huang H, Quek S. Comparison between karhunen-loeve and wavelet expansions for simulation of gaussian processes. Computers \& structures 2004;82(1314):985-91.

[33] Blacklock M, Bale H, Begley M, Cox B. Generating virtual textile composite specimens using statistical data from micro-computed tomography: 1D tow representations for the Binary Model. Journal of the Mechanics and Physics of Solids 2012;60(3):45170 .

[34] Rinaldi R, Blacklock M, Bale H, Begley M, Cox B. Generating virtual textile composite specimens using statistical data from micro-computed tomography: 3D tow representations. Journal of the Mechanics and Physics of Solids 2012;60(8):1561-81.

[35] Cox B, Bale H, Begley M, Blacklock M, Do B, Fast T, et al. Stochastic virtual tests for high-temperature ceramic matrix composites. Annual Review of Materials Research 2014;44:1-51.

[36] Przybyla C, Simmons J, Zawada L, Godar T, Bricker S, Jackson M, et al. Statistical characterization of SIC/SIC ceramic matrix composites at the filament scale with bayesian segmentation, hough transform feature extraction, and pair correlation statistics. In: SAMPE 2013 Proceedings: Education \& Green Sky - Materials Technology for a Better World. Long Beach, CA: SAMPE; 2013,.

[37] Czabaj M, Riccio M, Whitacre W. Numerical reconstruction of graphite/epoxy composite microstructure based on sub-micron resolution X-ray computed tomography. Composites Science and Technology 2014;105:174-82.

[38] Fast T, Scott A, Bale H, Cox B. Topological and euclidean metrics reveal spatially nonuniform structure in the entanglement of stochastic fiber bundles. Journal of Materials Science 2015;50(6):2370-98.

[39] Ko F. Preform fiber architecture for ceramic-matrix composites. Ceramic Bulletin 1989;68(2):401-14.

[40] Marshall D, Cox B. Integral textile ceramic structures. Annual Review of Materials Research 2008;38:425-43.

[41] Bale H, Blacklock M, Begley M, Marshall D, Cox B, Ritchie R. Characterizing threedimensional textile ceramic composites using synchrotron x-ray micro-computedtomography. Journal of the American Ceramic Society 2012;95(1):392-402.

[42] Vanaerschot A, Panerai F, Cassell A, Lomov S, Vandepitte D, Mansour N. Uncertainty quantification of a 3-d woven carbon fabric for the adept thermal protection system. Submitted to Composites Science and Technology 2016;. 
[43] Hexcel . HexForce G0986 SB 1200 - Product Data Hexcel. 2014.

[44] Sudret B, Kiureghian AD. Stochastic finite element methods and reliability: a stateof-the-art report. Department of civil \& environmental engineering, University of California Berkeley; 2000.

[45] Jolliffe I. Principal Component Analysis. Berlin: Springer-Verlag; 1986.

[46] Arnst M, Ghanem R, Soize C. Identification of bayesian posteriors for coefficients of chaos expansions. Journal of Computational Physics 2010;229(9):3134-54.

[47] Vořechovský M, Novák D. Correlation control in small-sample monte carlo type simulations 1: A simulated annealing approach. Probabilistic Engineering Mechanics 2009;24(3):452-62.

[48] Jiang Y, Chen X. Geometric and algebraic algorithms for modelling yarn in woven fabrics. Journal of the Textile Institute 2005;96(4):237-45.

[49] Lomov S, Verpoest I, Cichosz J, Hahn C, Ivanov D, Verleye B. Meso-level textile composites simulations: Open data exchange and scripting. Journal of Composite Materials 2014;48(5):621-37.

[50] Lomov S, Ivanov D, Verpoest I, Zako M, Kurashiki T, Nakai H, et al. Meso-FE modelling of textile composites: road map, data flow and algorithms. Composites Science and Technology 2007;67(9):1870-91.

[51] Verleye B, Croce R, Griebel M, Klitz M, Lomov S, Morren G, et al. Permeability of textile reinforcements: Simulation, influence of shear and validation. Composites Science and Technology 2008;68(13):2804-10. 